\title{
Tuning Random Forests for Causal Inference Under Cluster-Level Unmeasured Confounding
}

\author{
Youmi Suk ${ }^{* 1}$ and Hyunseung Kang ${ }^{\dagger 2}$ \\ ${ }^{1}$ School of Data Science, University of Virginia \\ ${ }^{2}$ Department of Statistics, University of Wisconsin-Madison
}

October 1, 2021

\begin{abstract}
Recently, there has been growing interest in using machine learning methods for causal inference due to their automatic and flexible ability to model the propensity score and the outcome model. However, almost all the machine learning methods for causal inference have been studied under the assumption of no unmeasured confounding and there is little work on handling omitted/unmeasured variable bias. This paper focuses on a machine learning method based on random forests known as Causal Forests and presents five simple modifications for tuning Causal Forests so that they are robust to cluster-level unmeasured confounding. Our simulation study finds that adjusting the default tuning procedure with the propensity score from fixed effects logistic regression or using variables that are centered to their cluster means produces estimates that are more robust to cluster-level unmeasured confounding. Also, when these parametric propensity score models are mis-specified, our modified machine learning methods remain robust to bias from cluster-level unmeasured confounders compared to existing parametric approaches based on propensity score weighting. We conclude by demonstrating our proposals in a real data study concerning the effect of taking an eighth-grade algebra course on math achievement scores from the Early Childhood Longitudinal Study.
\end{abstract}

Keywords: Causal inference, Machine learning methods, Unmeasured variables, Omitted variable bias, Fixed effects models

(

\section{Introduction}

Recently, there has been increasing interest in using machine learning (ML) methods to estimate conditional average treatment effects (CATE) as well as average treatment effects (ATE) (Athey \& Imbens, 2016; Dorie et al., 2019; Hill, 2011; Imai \& Ratkovic, 2013, Künzel et al., 2019; Su et al., 2009; Suk et al., 2020; Wager \& Athey, 2018) because they can flexibly and (nearly) automatically fit the treatment model and/or the outcome model. Often, ML-based methods

*eub6uw@virginia.edu

†hyunseung@stat.wisc.edu

This article has been accepted for publication in Multivariate Behavioral Research, published by Taylor \& Francis. 
in causal inference have been developed under the assumption that all the confounders are measured. Not surprisingly, when unmeasured confounders are present, there is no guarantee that the ML methods produce consistent estimates of treatment effects. Although no method can eliminate biases arising from an arbitrary unmeasured confounder, the goal of this paper is to propose a set of simple and quick modifications to existing ML methods so that they are more robust against cluster-level unmeasured confounders.

Cluster-level confounders are a type of covariate affecting both the treatment and the outcome variables and are also shared within a cluster; they differ from individual-level confounders which are not shared between individuals. Cluster-level confounders are common in the social sciences where units are naturally clustered together and form hierarchical structures. For example, consider the kindergarten cohort of the Early Childhood Longitudinal Study (ECLS-K), a multilevel observational study which we use in later sections to demonstrate our methods. Suppose we are interested in studying the causal effect of students taking an eighth-grade algebra course on their math achievement scores; here, the treatment variable is assigned at the individual level (i.e., the student level), not at the cluster level (i.e., the school level). Algebra courses are mathematics courses offered inside a formal school system and previous studies have recommended policies that encourage more students to learn algebra before entering high school (Rickles, 2013). Possible individual-level confounders in our analysis include students' prior achievement scores and socio-economic status. Possible cluster-level confounders include the type of school (e.g., private or public), school funding, school location (e.g., urban, suburban, medium size city, small town), and school climate. In particular, prior studies suggested that school-level characteristics play an important role in students' propensity to take math courses and their performance on achievement measures (Anderson \& Chang, 2011; Cogan et al., 2001; Opdenakker \& Van Damme, 2001). Anderson and Chang (2011) showed that students from rural schools generally earn fewer mathematics credits than students from non-rural schools. Additionally, students from rural schools tend to enter high school at a slightly lower achievement level. Opdenakker and Van Damme (2001) showed that school composition (e.g., proportions of girls, mean socio-economic status) and school processes (e.g., school climate) have important main and interaction effects on students' achievement even after their initial abilities are accounted for. Cogan et al. (2001) also found that learning opportunities for mathematics in schools depended on the location of school, school size, and percentage of minority enrollment. Unfortunately, the ECLS-K data did not measure all these school-level characteristics. The data measured some school-level confounders, such as the location and the type of school, but it did not measure others, such as school funding of math instruction and emphasis on mathematics education. Thus, these unmeasured cluster-level confounders are a potential threat to consistently estimating the causal effect of interest.

Many prior works have studied how to deal with cluster-level unmeasured confounders in multilevel/multisite observational data, most notably by using fixed effects models and random effects models (Arpino \& Mealli, 2011; Firebaugh et al., 2013, Li et al., 2013 Li et al., 2019 Schuler et al., 2016). A common theme in these works is that they make strong assumptions about the distribution of the unmeasured cluster-level confounder or the relationship between the unmeasured confounder and the treatment (see section below on 'Propensity Score Weighting Estimators, for a detailed review). Some recent works go beyond the fixed and random effects models by using calibration (Yang, 2018), sufficient statistics for logistic propensity score models (He, 2018), generalized fixed effects models (Arkhangelsky \& Imbens, 2019), and grouping propensity scores (Lee et al., 2021) to estimate treatment effects in the presence of unmeasured cluster-level confounders. Finally, Zetterqvist et al. (2016) proposed a doubly robust approach, called the doubly robust conditional generalized estimating equation (DRCGEE) estimator. Specifically, the DRCGEE estimator uses a cluster-centering approach to remove cluster-constant components, including unobserved cluster-level confounders. The DRCGEE estimator is consistent for the treatment effect as long as either the propensity score or 
the outcome model is correctly specified. Additionally, under some assumptions, the estimator is optimal in terms of semiparametric efficiency (see section below on "Doubly Robust Conditional Generalized Estimating Equation [DRCGEE] Estimator" for a detailed review).

Unfortunately, all of the aforementioned methods require parametric models, either for the outcome variable or the treatment variable. Often, these parametric models are used out of convenience and may be difficult to justify, especially when there is insufficient scientific knowledge about the relationship between variables. Recent work by Suk and Kang (2020) proposed non-parametric approaches to estimate the ATE and the CATE under cluster-level unmeasured confounding. Specifically, the work proposed three new approaches, the first based on the usual doubly robust estimator of the ATE, the second based on using cluster-demeaned variables for both the treatment and outcome models, and the third based on combining the two approaches. Although the proposed methods show great promise in dealing with cluster-level unmeasured confounding, they cannot be directly used to re-tune or re-fit existing ML methods so that they are robust to cluster-level unmeasured confounders. In particular, these aforementioned approaches require writing a new loss function inside existing ML methods and solving for the minimum of this loss function, both of which may be non-trivial in practice.

The goal of this paper is to study simple modifications to existing ML-based methods so that they become more robust to cluster-level unmeasured confounding. The main advantage of this approach is that one can take software or a ML method "off-the-shelf" and adjust only the inputs so that it produces more robust estimates of the treatment effect in the presence of cluster-level unmeasured confounders. Our paper focuses on one popular ML method for causal inference based on random forests called Causal Forests (Athey et al., 2019; Wager \& Athey, 2018). We propose five simple adjustments to make Causal Forests more robust against unmeasured cluster-level confounders in multilevel observational studies (see section below on 'Causal Forests Under Cluster-Level Unmeasured Confounding' for details). We compare how well these modified Causal Forests perform compared to the default Causal Forests without any modifications. We also compare our modified Causal Forests to existing parametric-based methods. Our simulation studies vary many parameters, notably the sample size, the outcome type, the working model of the propensity score, the distribution of unmeasured cluster-level confounders, and the magnitudes of the effect from unmeasured cluster-level confounders. We find that two of our modifications are the only methods that remain robust throughout all the scenarios. We conclude by revisiting the empirical example above on analyzing the effect of taking an 8th grade algebra course on math achievement scores using the proposed methods.

\section{Potential Outcomes and Identification Under Cluster- Level Unmeasured Confounding}

We use the potential outcomes framework (Neyman, 1923 Rubin, 1974) and its extension to multilevel/clustered data (Hong \& Raudenbush, 2006) to define causal effects. Let $Z_{i j} \in\{0,1\}$ be a binary treatment variable where $Z_{i j}=1$ denotes that individual $i$ in cluster $j$ was treated and $Z_{i j}=0$ denotes that individual $i$ in group $j$ was untreated or received the control. Let $Y_{i j}(1)$ be the potential outcome if individual $i j$ were treated $\left(Z_{i j}=1\right)$, and let $Y_{i j}(0)$ be the potential outcome if individual $i j$ were untreated $\left(Z_{i j}=0\right)$. The observed outcome of individual $i j$ is denoted as $Y_{i j}$. Also, for every individual $i j, \mathbf{X}_{i j}$ denotes their measured individuallevel confounders, $\mathbf{W}_{j}$ denotes their measured cluster-level confounders, and $\mathbf{U}_{j}$ denotes their unmeasured cluster-level confounders.

Using the potential outcomes framework, we define the ATE as the average linear contrast between potential outcomes under treatment and control:

$$
\tau=E\left[Y_{i j}(1)-Y_{i j}(0)\right]
$$


For example, in our real data study, the ATE represents the overall effect of taking an eighthgrade algebra course on math achievement scores. The usual working assumptions to identify the ATE are (Hernan \& Robins, 2020; Imbens \& Rubin, 2015):

$$
\text { Stable Unit Treatment Value Assumption (SUTVA) }
$$

$$
Y_{i j}=Z_{i j} Y_{i j}(1)+\left(1-Z_{i j}\right) Y_{i j}(0)
$$

$$
\begin{aligned}
& \text { Conditional Ignorability: } Y_{i j}(1), Y_{i j}(0) \perp Z_{i j} \mid \mathbf{X}_{i j}, \mathbf{W}_{j}, \mathbf{U}_{j} \\
& \text { Positivity: } 0<e_{i j}<1 \text { where } e_{i j}:=P\left(Z_{i j}=1 \mid \mathbf{X}_{i j}, \mathbf{W}_{j}, \mathbf{U}_{j}\right)
\end{aligned}
$$

Briefly, (A1) (abbreviated as SUTVA) states that each individual's potential outcomes do not depend on others' treatment assignments and there is only one version of the treatment. In our empirical example, (A1) implies that (a) each student's potential outcomes $Y_{i j}(1)$ and $Y_{i j}(0)$ are not affected by his peers' treatment status and (b) there is a single version of the algebra course in the study. (A2) states that within every value of the confounders $\mathbf{X}_{i j}, \mathbf{W}_{j}, \mathbf{U}_{j}$, the treatment $Z_{i j}$ is conditionally independent of the potential outcomes $Y_{i j}(1)$ and $Y_{i j}(0)$. In our empirical example, (A2) implies that students with identical confounders are equally likely to take an algebra course. (A3) states that within each value of the confounders, the probability of receiving treatment, also known as the propensity score $e_{i j}$, is strictly between zero and one. In our empirical example, (A3) implies that a student's probability of taking the algebra course is not exactly equal to 0 or 1 . (A2) and (A3) are jointly referred to as strong ignorability (Rosenbaum \& Rubin, 1983).

We make a few additional remarks about Assumptions (A1)-(A3). First, if $\mathbf{U}_{j}$ were unmeasured, the ATE can still be identified from observed data by comparing the outcomes of treated and untreated groups within the same cluster and aggregating the within-cluster differences across all the clusters. Intuitively, this identification strategy works because cluster-level unmeasured confounders are the same for every study unit within each cluster $j$ and by (A2), the treatment would remain conditionally independent of the potential outcomes within a cluster. For more details on identification under cluster-level unmeasured confounding, see Kim and Steiner $(2020)$ and Imai and Kim $(2019)$ for a graphical perspective and Arkhangelsky and Imbens (2019) for a potential outcomes perspective. Second, (A2) and (A3) allow for unmeasured cluster-level confounders and if most of the bias is from cluster-level unmeasured confounders, the methods we present below will provide a less biased estimate of the causal effect than methods that assume no unmeasured confounding of any kind. Of course, if individual-level unmeasured confounders are present and the bias from them is strong, the methods we present below will just be as biased as the methods that assume no unmeasured confounding of any kind and a sensitivity analysis would be more appropriate to analyze the presence (or absence) of a causal effect. Even then, methods that account for cluster-level unmeasured confounders may be less sensitive to unobserved biases compared to methods that assume no unmeasured confounding of any kind (Suk \& Kang, 2020).

\section{Existing Estimation Methods Under Unmeasured Cluster-Level Confounding}

In the next two subsections, we present a review of some popular estimation methods to estimate the ATE in multilevel observational studies with cluster-level unmeasured confounding. The first subsection primarily focuses on methods based on the propensity score (Arpino \& Mealli, 2011; Hong \& Raudenbush, 2006; Kim \& Seltzer, 2007; Leite et al., 2015, Li et al., 2013; Rickles \& Seltzer, 2014 Schuler et al., 2016; Steiner et al., 2012; Thoemmes \& West, 2011), and the

For relaxing SUTVA in multilevel settings, see Hong and Raudenbush (2006) and Hong and Raudenbush (2013). 
next subsection summarizes a recent approach called the DRCGEE estimator (Zetterqvist et al., 2016) that uses both the outcome and propensity score models to obtain a doubly robust estimator of the ATE.

\subsection{Propensity Score Weighting Estimators}

Broadly speaking, propensity score weighting estimators can be broken down into two steps: (i) choosing/estimating a propensity score and (ii) using the estimated propensity score as sampling weights to estimate the ATE. For the first step, most propensity score models in multilevel observational studies fall into two types: within-cluster propensity score models and across-cluster (or between-cluster) propensity score models. Within-cluster propensity score models are separate cluster-specific models of the selection process. Across-cluster propensity score models use a single model for the selection process for all the clusters and often include cluster-level random effects or fixed effects (Arpino \& Mealli, 2011; Leite et al., 2015; Li et al., 2013. Schuler et al., 2016). Between the two types, within-cluster propensity score models are the most flexible, but difficult to estimate when (i) strong treatment selection processes are present, (ii) cluster sizes are small, and (iii) there are no treated units or no control units within each cluster, indicating a potential violation of Assumption (A3) (Kim \& Seltzer, 2007; Leite et al., 2015; Steiner et al., 2012 Thoemmes \& West, 2011). Across-cluster propensity score models alleviate these concerns at the expense of making additional assumptions about the selection process. Specifically, fixed effects and random effects propensity score models used in across-cluster propensity score models usually take on the following forms:

$$
\begin{aligned}
\text { Fixed Effects: } \operatorname{logit}\left(e_{i j}\right) & =\mu_{j}+\mathbf{X}_{i j}^{\top} \boldsymbol{\alpha} \\
\text { Random Effects: } \operatorname{logit}\left(e_{i j}\right) & =\mu_{j}+\mathbf{X}_{i j}^{\top} \boldsymbol{\alpha}, \quad \mu_{j} \sim N\left(\mu_{0}, \sigma^{2}\right)
\end{aligned}
$$

In both fixed and random effects propensity scores, the term $\mu_{j}$ describes the effects of cluster-

level covariates on the treatment and the term $\mathbf{X}_{i j}^{\top} \boldsymbol{\alpha}$ describes the effect of measured individuallevel covariates on the treatment. Fixed effects models assume that $\mu_{j}$ is fixed and are represented by cluster dummy variables that indicate individual $i j$ 's membership in cluster $j$ ( $\mathrm{Li}$ et al., 2013 Wooldridge, 2010). In contrast, random effects models assume that $\mu_{j}$ is normally distributed with mean $\mu_{0}$ and common variance $\sigma^{2}$. Additionally, $\mu_{j}$ is assumed to be independent of observed covariates. Random effects models can also include measured cluster-level covariates; fixed effects models are not identified if there are cluster-level covariates. Both propensity score models are usually estimated by using the maximum likelihood principle. In particular, random effects propensity score models are often discussed in conjunction with Bayesian shrinkage estimation where the magnitude of shrinkage is determined by each cluster's reliability and cluster size (Li et al., 2013, Raudenbush \& Bryk, 2002).

A major strength of fixed effects models is that they allow for arbitrary correlation between unobserved cluster effects and the observed covariates (Li et al., 2013; Wooldridge, 2010). However, including cluster dummy variables increases the dimension of the propensity score model and can potentially cause numerical instability when there are many small clusters (Li et al., 2013 Snijders \& Bosker, 2012). In contrast, random effects models impose a parametric, distributional assumption on $b_{j}$ to reduce the number of parameters for estimation and alleviate some numerical issues when there are many small clusters. But the additional assumptions made in random effects models may not hold in practice. For more discussions on random effects and fixed effects propensity score models, see Arpino and Mealli (2011), Snijders and Bosker (2012), Li et al. (2013), and Schuler et al. (2016).

Once an appropriate propensity score model has been selected and estimated, the next step is to estimate the treatment effect. Often, this is done via some form of sample reweighing where the estimated propensity score for each person is used as an inverse probability weight (IPW). 
The goal of IPW is to achieve covariate balance between the treatment group and the control group. For alternative methods that use the estimated propensity score based on matching and stratification, see Schafer and Kang (2008), Stuart (2010), and Lunceford and Davidian (2004). Broadly speaking, there are two types of IPW estimators for estimating the ATE in multilevel observational studies with unmeasured cluster-level confounding: the marginal IPW estimator and the clustered IPW estimator. A marginal IPW estimator, denoted as $\hat{\tau}^{\mathrm{MIPW}}$, is an acrosscluster comparison estimator (Leite, 2016; Steiner et al., 2012; Thoemmes \& West, 2011) where we compute a weighted difference between the overall outcome mean among the treated units and the control units and the weights are the inverse of the estimated propensity scores:

$$
\hat{\tau}^{\mathrm{MIPW}}=\frac{\sum_{j=1}^{J} \sum_{i=1}^{n_{j}} Z_{i j} w_{i j} Y_{i j}}{\sum_{j=1}^{J} \sum_{i=1}^{n_{j}} Z_{i j} w_{i j}}-\frac{\sum_{j=1}^{J} \sum_{i=1}^{n_{j}}\left(1-Z_{i j}\right) w_{i j} Y_{i j}}{\sum_{j=1}^{J} \sum_{i=1}^{n_{j}}\left(1-Z_{i j}\right) w_{i j}}, w_{i j}=\frac{Z_{i j}}{e_{i j}}+\frac{1-Z_{i j}}{\left(1-e_{i j}\right)}
$$

The clustered IPW estimator, denoted as $\hat{\tau}^{\mathrm{CIPW}}$, is a within-cluster comparison estimator (Leite, 2016 Steiner et al., 2012; Thoemmes \& West, 2011) where we compute a weighted average of cluster-specific ATEs (Li et al., 2013):

$$
\hat{\tau}^{\mathrm{CIPW}}=\frac{\sum_{j=1}^{J} w_{j} \hat{\tau}_{j}}{\sum_{j=1}^{J} w_{j}}, \hat{\tau}_{j}=\frac{\sum_{i=1}^{n_{j}} Z_{i j} w_{i j} Y_{i j}}{\sum_{i=1}^{n_{j}} Z_{i j} w_{i j}}-\frac{\sum_{i=1}^{n_{j}}\left(1-Z_{i j}\right) w_{i j} Y_{i j}}{\sum_{i=1}^{n_{j}}\left(1-Z_{i j}\right) w_{i j}}, w_{j}=\sum_{i=1}^{n_{j}} w_{i j}
$$

Comparing the marginal and the clustered IPW estimators, the marginal IPW estimator does not always guarantee covariate balance within clusters because the averages are taken across clusters instead of within clusters (Li et al., 2013; Thoemmes \& West, 2011). In contrast, the clustered IPW estimator with a correct propensity score model achieves covariate balance within clusters; it also allows investigators to estimate cluster-specific ATEs. But, the clustered IPW estimator requires each cluster to have at least one treated and one control unit to have a meaningful estimate of $\hat{\tau}_{j}$, whereas the marginal IPW estimator does not have this requirement. $\mathrm{Li}$ et al. (2013) showed that under a balanced design where there is at least one treated and one control unit per cluster, the marginal IPW estimator with a fixed effects propensity score model is numerically equivalent to the clustered IPW estimator with a fixed effects propensity score model. Finally, we remark that there are hybrid methods that combine elements of the across-cluster and within-cluster comparison estimators, such as two-stage matching (Rickles \& Seltzer, 2014), preferential matching (Arpino \& Cannas, 2016), and within-class matching (Kim \& Steiner, 2015).

Unfortunately, all the aforementioned propensity score methods require specifying a parametric model for the propensity score. Although this requirement may be reasonable in some cases, these models are often chosen out of convenience or simplicity and there is often insufficient justification for the plausibility of these models.

\subsection{Doubly Robust Conditional Generalized Estimating Equa- tion (DRCGEE) Estimator}

Recently, Zetterqvist et al. (2016) proposed a new method to estimate the ATE in multilevel observational studies with unmeasured cluster-level confounding by using both the outcome and the propensity score models. Specifically, consider the following model for the outcome and the treatment:

$$
E\left[Y_{i j} \mid Z_{i j}, \mathbf{X}_{i j}, \mathbf{W}_{j}\right]=a_{j}+Z_{i j} \mathbf{V}_{i j}^{\top} \boldsymbol{\beta}+\mathbf{X}_{i j}^{\top} \boldsymbol{\gamma}, E\left[Z_{i j} \mid \mathbf{X}_{i j}, \mathbf{W}_{j}\right]=b_{j}+\mathbf{X}_{i j}^{\top} \boldsymbol{\alpha}
$$

The terms $a_{j}$ and $b_{j}$ are the cluster-specific intercepts in the outcome and treatment models, respectively. The term $\mathbf{V}_{i j}$ contains observed covariates that interact with the treatment and the 
term $\boldsymbol{\beta}$ represents the treatment effect coefficient. The terms $\boldsymbol{\gamma}$ and $\boldsymbol{\alpha}$ are regression coefficients of measured individual-level covariates in the outcome model and the treatment model, respectively. For known $\boldsymbol{\gamma}$ and $\boldsymbol{\alpha}$, the DRCGEE estimator estimates the treatment effect coefficient $\boldsymbol{\beta}$ by solving the following estimating equation:

$$
\mathbf{0}=\sum_{i j} \mathbf{V}_{i j}\left(S_{Z, i j}-\bar{S}_{X, j}\right)\left(S_{Y, i j}-\bar{S}_{Y, j}\right), S_{Y, i j}=Y_{i j}-Z_{i j} \mathbf{V}_{i j}^{\top} \boldsymbol{\beta}-\mathbf{X}_{i j}^{\top} \boldsymbol{\gamma}, S_{Z, i j}=Z_{i j}-\mathbf{X}_{i j}^{\top} \boldsymbol{\alpha}
$$

The structure of the above estimating equation, specifically the product of the two terms in parentheses, makes the DRCGEE estimator doubly robust, i.e., the estimator is consistent for the treatment effect if either the propensity score model or the outcome model is correctly specified. As noted by Zetterqvist et al. (2016), under some settings, the DRCGEE estimator is identical to the conditional generalized estimating equation (CGEE) estimator (Goetgeluk \& Vansteelandt, 2008), the standard G-estimator (Robins et al., 1992), or the ordinary least squares estimator in linear regression. For example, when there are no measured confounders, but there are cluster-level unmeasured confounders, $\beta$ in equation (6) can be found by running a fixed effects linear regression model with the outcome $Y_{i j}$ and the regressor $Z_{i j}$. In other words, the DRCGEE estimator can be seen as a generalization of the fixed effects linear regression model, a generalization that allows for double robustness. Also, while not mentioned in Zetterqvist et al. (2016), we remark that another way to estimate $\beta$ in equation (6) is by fitting an instrumental variable regression based on generalized method of moments (Hansen, 1982) where the instrumental variables are the product of $\mathbf{V}_{i j}$ and the residualized cluster-demeaned treatment, the endogenous variables are cluster-demeaned variables $Z_{i j} \mathbf{V}_{i j}$, the outcome variable is the residualized cluster-demeaned outcome, and the weighting matrix is an identity matrix (Suk \& Kang, 2020). Moreover, under some additional assumptions, Zetterqvist et al. (2016) showed that the DRCGEE estimator is optimal in terms of semiparametric efficiency. Finally, we note that if the treatment model is based on a logistic regression model, the DRCGEE estimator uses a conditional logistic regression (Zetterqvist et al., 2016).

Although the DRCGEE estimator improves upon the propensity score estimators in the prior subsection by being doubly robust, the DRCGEE estimator still requires parametric models for the outcome or the treatment. As mentioned earlier, researchers could potentially misspecify both of these models and doubly robust estimators can perform worse than non-doubly robust methods when both models are mis-specified (Kang \& Schafer, 2007).

\section{Causal Forests Under Cluster-Level Unmeasured Confounding}

\subsection{Default Causal Forests}

In this section, we briefly review the Causal Forest estimator of the CATE $\tau(\mathbf{x}, \mathbf{w})$ when clusterlevel confounders are absent. Let $m(\mathbf{x}, \mathbf{w})=E\left[Y_{i j} \mid \mathbf{X}_{i j}=\mathbf{x}, \mathbf{W}_{i j}=\mathbf{w}\right]$ be the outcome regression and $e(\mathbf{x}, \mathbf{w})=E\left[Z_{i j} \mid \mathbf{X}_{i j}=\mathbf{x}, \mathbf{W}_{i j}=\mathbf{w}\right]$ be the propensity score. Broadly speaking, the Causal Forests estimator is based on a weighted linear regression of a residualized outcome $\tilde{Y}_{i j}=Y_{i j}-\hat{m}^{-i j}(\mathbf{x}, \mathbf{w})$ and a residualized regressor $\tilde{Z}_{i j}=Z_{i j}-\hat{e}^{-i j}(\mathbf{x}, \mathbf{w})$ with the weight $\alpha_{i j}(\mathbf{x}, \mathbf{w})$. The ${ }^{(-i j)}$-superscripts represent out-of-bag, leave-one-out estimates in machine learning, i.e., estimates without using study unit $i j$ 's data. Typically, Causal Forests uses an honest random forest algorithm to compute $\hat{m}^{-i j}(\mathbf{x}, \mathbf{w})$ and $\hat{e}^{-i j}(\mathbf{x}, \mathbf{w})$, although a consistent estimator with some statistical properties will suffice (see Section 6.1.1 of Athey et al., 2019 for the exact statistical properties). Here, honesty is a property of trees where the data used to determine the 
structure of the tree is not used again to estimate the treatment effect within each leaf of the tree. Procedure 1 in Wager and Athey (2018) returns an honest tree by subsampling the data into two halves: One for estimating the structure of the tree, specifically when and where to split the tree to create leaves of the tree, and the other to estimate the treatment effect within each leaf of the tree. Once $m$ and $e$ are estimated, the Causal Forest estimator of the CATE can be written as:

$$
\hat{\tau}(\mathbf{x}, \mathbf{w})=\frac{\sum_{i j} \alpha_{i j}(\mathbf{x}, \mathbf{w})\left(Y_{i j}-\hat{m}^{(-i j)}\left(\mathbf{X}_{i j}, \mathbf{W}_{j}\right)\right)\left(Z_{i j}-\hat{e}^{(-i j)}\left(\mathbf{X}_{i j}, \mathbf{W}_{j}\right)\right)}{\sum_{i j} \alpha_{i j}(\mathbf{x}, \mathbf{w})\left(Z_{i j}-\hat{e}^{(-i j)}\left(\mathbf{X}_{i j}, \mathbf{W}_{j}\right)\right)^{2}}
$$

The terms $\alpha_{i j}(\mathbf{x}, \mathbf{w}), 0 \leq \alpha_{i j}(\mathbf{x}, \mathbf{w}) \leq 1$, weigh how much each data point $i j$ contributes to the estimate of CATE by measuring how far $i j$ 's covariates $\mathbf{X}_{i j}, \mathbf{W}_{j}$, are away from $\mathbf{x}, \mathbf{w}$; a large $\alpha_{i j}(\mathbf{x}, \mathbf{w})$ means that data point $i j$ provides a large contribution to $\hat{\tau}(\mathbf{x}, \mathbf{w})$, and a small $\alpha_{i j}(\mathbf{x}, \mathbf{w})$ means that data point $i j$ provides a small contribution to $\hat{\tau}(\mathbf{x}, \mathbf{w})$. Estimating the weights $\alpha_{i j}(\mathbf{x}, \mathbf{w})$ is based on subsampling and a recursive partitioning algorithm popular in tree-based ML algorithms and Athey et al. (2019) provides additional details. Finally, under some assumptions, notably Assumptions 1 through 6 in Athey et al. (2019) plus a rate condition on the tree's asymptotic size relative to the subsamples used to create the leaves, the Causal Forests estimates are consistent and asymptotically normal.

Like most ML methods, Causal Forests can be fine-tuned to improve its performance. For example, investigators can manipulate the minimum node size of each tree and change the penalty for imbalanced splits. More recently, Suk et al. (2020) augmented Causal Forests with multilevel propensity scores from random effects logistic regression by replacing the default estimator of $\hat{e}^{(-i j)}$ in order to improve its performance in multilevel observational data under no unmeasured confounding. The vignette of the $\mathrm{R}$ ( $\mathrm{R}$ Core Team, 2020) software grf (Tibshirani et al., 2020) provides other ways of fine-tuning Causal Forests. In this article, we propose modifications to Causal Forests to make it more robust to cluster-level unmeasured confounding.

\subsection{Modifications for Cluster-Level Unmeasured Confounding}

We study five simple modifications to make Causal Forests more robust to cluster-level unmeasured confounders (see Table 1 for a summary). The first modification, denoted as CF+RePS, forces Causal Forests to use propensity scores from random effects logistic regression as $\hat{e}^{(-i j)}$; this is done by using the parameter W. hat in the grf package. The second modification, denoted as $\mathrm{CF}+\mathrm{FePS}$, forces Causal Forests to use propensity scores from fixed effects logistic regression as $\hat{e}^{(-i j)}$; this is, again, done by using the parameter W.hat in the grf package. These two modifications are motivated from (i) Section 'Propensity Score Weighting Estimators' where unobserved cluster-specific effects are modeled as random effects or fixed effects and (ii) the underlying implementation of the Causal Forest estimator, especially when it comes to averaging across individuals. As noted in equation (8) and Section 2.1 of Athey and Wager (2019), Causal Forests uses a doubly robust score to aggregate CATE estimates across all participants in the sample in order to estimate the ATE:

$$
\begin{aligned}
\hat{\tau} & =\frac{1}{n} \sum_{i j} \hat{\tau}_{i j}, \\
\hat{\tau}_{i j} & =\hat{\tau}^{(-i j)}\left(\mathbf{X}_{i j}, \mathbf{W}_{j}\right) \\
& +\frac{Z_{i j}-\hat{e}^{(-i j)}\left(\mathbf{X}_{i j}, \mathbf{W}_{j}\right)}{\hat{e}^{(-i j)}\left(\mathbf{X}_{i j}, \mathbf{W}_{j}\right)\left(1-\hat{e}^{(-i j)}\left(\mathbf{X}_{i j}, \mathbf{W}_{j}\right)\right)}\left(Y_{i j}-\hat{m}^{(-i j)}\left(\mathbf{X}_{i j}, \mathbf{W}_{j}\right)-\left(Z_{i j}-\hat{e}^{(-i j)}\left(\mathbf{X}_{i j}, \mathbf{W}_{j}\right)\right) \hat{\tau}\left(\mathbf{X}_{i j}, \mathbf{W}_{j}\right)^{(-i j)}\right) .
\end{aligned}
$$

Similar to $\hat{e}^{(-i j)}$ and $\hat{m}^{(-i j)}, \hat{\tau}^{(-i j)}$ are out-of-bag estimates that do not include individual $i j$ 's data. The double robustness of Causal Forests arises from the middle term following $\hat{\tau}^{(-i j)}\left(\mathbf{X}_{i j}, \mathbf{W}_{j}\right)$ where a correctly specified propensity score can lead to a consistent estimate 
of the CATE, irrespective of the outcome model. Section 5 of Nie and Wager $(2020)$ and Sections 4 and 5 of Kennedy (2020) provide the exact conditions under which this result holds; these conditions involve smoothness of both the propensity score and the outcome model. In practical terms, if the fixed or the random effects propensity score models eliminate the impact of cluster-level unmeasured confounders, using them as $\hat{e}^{(-i j)}$ will lead to consistent estimators of the average treatment effect. But, recall that the propensity score models are parametric models, models which can be mis-specified and lead to bias. As we show in the simulations below, even if they are partially misspecified, the resulting Causal Forest estimates remain robust compared to their IPW counterparts due, in part, to the flexible modeling of the outcome regression $m^{(-i j)}$ (double robustness).

Table 1: Summary of modifications in Causal Forests

\begin{tabular}{|c|c|c|}
\hline Modification & Description & $\begin{array}{l}\text { Parameter(s) to } \\
\text { modify in grf }\end{array}$ \\
\hline $\mathrm{CF}+\mathrm{RePS}$ & $\begin{array}{l}\text { Add propensity scores from random effects logistic } \\
\text { regression }\end{array}$ & W.hat \\
\hline $\mathrm{CF}+\mathrm{FePS}$ & $\begin{array}{l}\text { Add propensity scores from fixed effects logistic } \\
\text { regression }\end{array}$ & W.hat \\
\hline $\mathrm{CF}+$ ClusterID & Add cluster dummies as covariates & $x$ \\
\hline $\mathrm{CF}+$ Demean & $\begin{array}{l}\text { Use demeaned outcome, demeaned treatment, and } \\
\text { demeaned and cluster-constant components of } \\
\text { covariates }\end{array}$ & $\mathrm{Y}, \mathrm{W}, \mathrm{X}$ \\
\hline \multirow[t]{2}{*}{$\mathrm{CF}+$ Demean $+\mathrm{PS}$} & $\begin{array}{l}\text { Use demeaned outcome, demeaned treatment, } \\
\text { demeaned and cluster-constant components of } \\
\text { covariates }\end{array}$ & $\mathrm{Y}, \mathrm{W}, \mathrm{X}$ \\
\hline & $\begin{array}{l}\text { Add demeaned propensity scores based on demeaned } \\
\text { variables }\end{array}$ & W.hat \\
\hline
\end{tabular}

Note: grf is an $\mathrm{R}$ package to implement Causal Forests. Demeaned variables represent variables that are centered around their cluster means.

The third modification, denoted as CF+ClusterID, includes cluster dummy variables as part of the covariates. The motivation behind this modification is that Causal Forests will use the cluster dummy variables to non-parametrically adjust for unobserved cluster-level differences between the treated and control groups when it fits $e^{(-i j)}$ and $m^{(-i j)}$ using regression forests. A bit more formally, as shown in Suk and Kang, 2020, irrespective of the functional form of the outcome or the propensity score model, all cluster-level unmeasured confounders $\mathbf{U}_{j}$ must be an unknown linear function of cluster dummy variables:

$$
\mathbf{U}_{j}=f_{\boldsymbol{\Gamma}}\left(\mathbf{D}_{i j}\right)=\mathbf{D}_{i j} \boldsymbol{\Gamma} .
$$

Here, $\boldsymbol{\Gamma}$ denotes a $J$ by $p_{u}$ matrix of parameters where $p_{u}$ is the dimension of the unmeasured cluster-level covariates. $\mathbf{D}_{i j} \in\{0,1\}^{J}$ denotes the $J$ dimensional cluster dummy variables where the $j$ th element equals 1 and the rest equal 0. $f$ denotes a linear function parametrized by $\boldsymbol{\Gamma}$. We can use $\mathbf{D}_{i j}$ as a proxy for $\mathbf{U}_{j}$ inside the outcome model and the propensity score model. Then, we can use flexible machine learning methods to estimate $\boldsymbol{\Gamma}$. For example, to train the propensity score $e_{i j}=P\left(Z_{i j}=1 \mid \mathbf{X}_{i j}, \mathbf{W}_{j}, \mathbf{U}_{j}\right)$ that depends on the unmeasured cluster-level confounder $\mathbf{U}_{j}$, we can use the relationship in equation (8) and arrive at

$$
e_{i j}=P\left(Z_{i j}=1 \mid \mathbf{X}_{i j}, \mathbf{W}_{j}, \mathbf{U}_{j}\right)=P\left(Z_{i j}=1 \mid \mathbf{X}_{i j}, \mathbf{W}_{j}, f_{\boldsymbol{\Gamma}}\left(\mathbf{D}_{i j}\right)\right)
$$

The right-hand-side of the above expression is a function of measured quantities, $\mathbf{X}_{i j}, \mathbf{W}_{j}$ and $\mathbf{D}_{i j}$, and we can treat $\boldsymbol{\Gamma}$ as an additional parameter to estimate when estimating the propensity 
score. That is, so long as we use a flexible ML-based method like Causal Forests to estimate the right-hand-side, we can approximate the term $\mathbf{D}_{i j} \boldsymbol{\Gamma}$ and consequently, approximate the original propensity score $e_{i j}$. We remark that this modification is comparable to including cluster fixed effects in linear or nonlinear models, except Causal Forests is a non-parametric method. A downside of this modification is that if there are many clusters, adding cluster dummy variables to the set of covariates may result in overfitting.

The fourth modification, denoted as CF+Demean, uses the cluster-demeaned outcome, cluster-demeaned treatment, cluster-demeaned measured confounders, and cluster-constant measured confounders as inputs to the grf package. Cluster-demeaned measured confounders are individual-level measured confounders where we subtract their cluster means, creating what we term demeaned variables. Cluster-constant measured confounders are cluster-level covariates (e.g., the location of school) and cluster-mean variables of individual-level covariates (e.g., the cluster means of student's prior achievement scores). The fourth modification is motivated by two observations, one from local linear regression and another from linear approximations of binary response models. First, suppose we only have $p_{x}$ individual-level covariates $\mathbf{X}_{i j}$ and there exists a subspace $\mathcal{X} \subseteq \mathbb{R}^{p_{x}}$ where the observed data can be locally approximated by a linear model, i.e.,

$$
E\left[Y_{i j} \mid Z_{i j}, \mathbf{X}_{i j}\right]=a_{j}+Z_{i j} \beta, \quad \text { if } \quad \mathbf{X}_{i j} \in \mathcal{X} .
$$

Subtracting the cluster means from the original observed variables creates variables that are locally orthogonal to cluster-specific variations in the original variables. Since unmeasured cluster-level confounders reside in the subspace consisting of cluster-specific variations, working with demeaned variables that are locally orthogonal to this subspace could help remove biases from cluster-level unmeasured confounders. Indeed, page 1169 of Athey et al. (2019) exploits this idea of local linearity of the outcome model to justify the robustness of their methods to measured confounders $\mathbf{X}_{i j}$. Second, under some assumptions, a propensity score model with binary treatment can be approximated by a linear regression model and thus, the propensity score can be treated as if it lies inside a linear subspace (Caliendo \& Kopeinig, 2008; Rubin \& Thomas, 1992; Wooldridge, 2010). The last modification, denoted as CF+Demean+PS, is an extension of the fourth modification using demeaned variables where we use the demeaned propensity score as $e^{(-i j)}$. A demeaned propensity score fits a linear regression with the demeaned treatment as the dependent variable and the demeaned first-order and higher-order terms of $\mathbf{W}_{j}$ and $\mathbf{X}_{i j}$ as the independent variables.

We make some additional remarks about the modifications. First, CF+ClusterID is different from $\mathrm{CF}+$ Demean because Causal Forests is a nonparametric model; if Causal Forests uses linear models for both the outcome and the treatment, $\mathrm{CF}+$ ClusterID and $\mathrm{CF}+$ Demean would be identical, up to the weighting term $\alpha_{i j}$. Second, none of the modifications explicitly require that the estimation methods of the propensity score model and the outcome model are identical. For example, the propensity score may follow a parametric logistic model, but the outcome model may use a nonparametric regression model. Third, there is no formal mathematical justification that the proposed modifications to Causal Forests will lead to consistent, asymptotically Normal estimates of the treatment effect under all arbitrary forms of cluster-level unmeasured confounding. But, as our simulation results show below, the proposed modifications hold promise in addressing with a wide variety of cluster-level unmeasured confounders; we believe under the right set of theoretical assumptions, the modified Causal Forests will have these favorable statistical properties. Fourth, the proposed modifications to Causal Forests can be viewed as an a priori across-cluster approach because they do not fit a separate model for each cluster. Specifically, Causal Forests aggregates multiple trees built from different re-samples of the entire dataset to estimate the ATE and hence, functions as an across-cluster approach. However, the adaptive weights $\alpha_{i j}$ in Causal Forests are often local weights that give higher weights to units that are closer to a particular value of the covariates and hence, could approximate a 
within-cluster approach. Also, using external information (e.g., fixed-effects propensity scores) or transformed variables (e.g., cluster-demeaned variables) allows ML methods to behave more similarly to a within-cluster approach (see our simulation results below).

\section{Simulation Study}

We conducted simulation studies to assess which of the proposed modifications improve the performance of Causal Forests in multilevel observational data with cluster-level unmeasured confounding. Throughout the simulations, we estimate the ATE using (1) our five modifications outlined above, (2) the default Causal Forests estimator without any modifications, (3) the DRCGEE estimator, and (4) the marginal and clustered IPW estimators under two types of propensity score models: a random effects logistic regression model and a fixed effects logistic regression model.

Our simulation studies are divided into four parts and summarized in Table 2. Design 1 uses a normally distributed unmeasured cluster-level confounder $U_{j}$ and a linear outcome model. Design 2 assumes a uniformly distributed $U_{j}$ and a linear outcome model. The first two designs are meant to assess the performance of random-effects-based estimators versus fixedeffects-based estimators because random effects models require an assumption that $U_{j}$ follows a normal distribution. Design 3 is based on Design 2, but uses a misspecified propensity score model by excluding higher-order terms; note that for the DRCGEE estimator, we misspecified the outcome model. In particular, Design 3 reflects a scenario whereby a careful researcher, despite his/her best efforts, was partially successful in correctly specifying the propensity score model (or the outcome model for the DRCGEE estimator). Design 4 is based on Design 2, but uses a nonlinear, binary outcome model instead of a linear outcome model. In Appendix A, we conducted additional simulations where the outcome model has exponential errors. More specifically, we used the same design as Design 2, except the term $r_{i j}$ in the outcome model follows an exponential distribution, i.e., $r_{i j} \sim \exp (1 / 5)$. The results of this additional design were similar to those from Design 2.

For each of the four designs, we vary the sample size and the magnitudes of the effects from unmeasured confounders. Combining all of the variations across the four designs (plus the additional variations in the Appendices), our simulation study considers 150 different data generating models. We examine the performance of the proposed methods in each data generating model. As we show below, unlike parametric-based methods, our ML-based methods are able to automatically adapt and flexibly model the underlying data generating model while controlling for biases from cluster-level unmeasured confounders.

Table 2: Summary of Simulation Designs 1, 2, 3, and 4

\begin{tabular}{lllll}
\hline & Design 1 & Design 2 & Design 3 & Design 4 \\
\hline $\begin{array}{l}\text { Distribution of } U_{j} \\
\text { Outcome Model }\end{array}$ & L $(0,1)$ & Uniform(-2, 2) & Uniform(-2, 2) & Uniform(-2, 2) \\
& & Linear & Linear & $\begin{array}{l}\text { Non-linear } \\
\text { (logistic) }\end{array}$ \\
$\begin{array}{l}\text { Higher-Order Terms } \\
\begin{array}{l}\text { Included in Estimated } \\
\text { Models }\end{array}\end{array}$ & Yes & Yes & No & Yes \\
\hline
\end{tabular}

For all simulation designs, we repeated the simulation 1,000 times, and we evaluated the performance of each estimator by measuring the percent bias (Bias(\%)), standard deviation 
(SD), and root mean squared error (RMSE) defined as follows:

$$
\begin{aligned}
\operatorname{Bias}(\%) & =100 \times\left|\frac{1}{1,000} \sum_{m=1}^{1,000} \frac{\left(\hat{\tau}_{m}-\tau\right)}{\tau}\right| \\
\mathrm{SD} & =\sqrt{\frac{1}{1,000-1} \sum_{m=1}^{1,000}\left(\hat{\tau}_{m}-\overline{\hat{\tau}}\right)^{2}} \\
\mathrm{RMSE} & =\sqrt{\frac{1}{1,000} \sum_{m=1}^{1,000}\left(\hat{\tau}_{m}-\tau\right)^{2}}
\end{aligned}
$$

Here, $\hat{\tau}_{m}, m=1, \ldots, 1,000$ is the $m$ th estimate of the ATE from 1,000 replications, and $\overline{\hat{\tau}}$ is the sample mean of $\hat{\tau}_{m}$ across 1,000 replications. R codes for our simulation study are available in the supplementary materials (DataGeneratingModels.R; SimulationCodes.R) and the first author's GitHub repository]

\subsection{Design 1: Normally Distributed $U_{j}$ and Linear Outcome Model}

The data generating models below are based on those from Arpino and Mealli (2011), Li et al. (2013), Schuler et al. (2016), and Zetterqvist et al. (2016).

1. For each cluster $j=1, \ldots, J$, generate the total number of individuals in each cluster $n_{j}$ by drawing a number from a normal distribution with mean $M$ and standard deviation $S$ and rounding it to the nearest integer. Each simulation consisted of roughly 4,000 individuals, and we used five sample size conditions of $\left(J, M_{S}\right):\left(400,10_{0.5}\right),\left(200,20_{1}\right),\left(100,40_{2}\right),\left(50,80_{2}\right)$, and $\left(20,200_{2}\right)$. We remark that $M$ increases by a factor of 2 , whereas $J$ decreases by a factor of 2, which keeps the total sample size approximately constant across the five conditions.

2. For each individual $i=1, \ldots, n_{j}$ in cluster $j$, generate individual-level confounders, $\mathbf{X}_{i j}=$ $\left(X_{1 i j}, X_{2 i j}, X_{3 i j}\right)$, a cluster-level measured confounder $W_{j}$, and a cluster-level unmeasured confounder $U_{j}$ as follows.

$$
\begin{gathered}
X_{1 i j} \sim \operatorname{Uniform}(-1,1), X_{2 i j} \sim N(0,1), X_{3 i j} \sim \operatorname{Uniform}(0,1), \\
W_{j} \sim \operatorname{Uniform}(-1,1), U_{j} \sim N(0,1)
\end{gathered}
$$

3. Generate individual treatment status $Z_{i j}$ from the logistic propensity score model below.

$$
\begin{aligned}
\operatorname{logit}\left(e_{i j}\right) & =-0.6+0.3 X_{1 i j}+0.3 X_{2 i j}+0.3 X_{3 i j}+0.3 W_{j}+0.4 X_{1 i j}^{2}+0.4 X_{2 i j} W_{j} \\
& +0.4 X_{1 i j} I\left(X_{3 i j}<0.3\right)+0.3 U_{j} \\
Z_{i j} & \sim \text { Bernoulli }\left(e_{i j}\right)
\end{aligned}
$$

The propensity score model contains a quadratic term (i.e., $X_{1 i j}^{2}$ ), an interactive, nonlinear threshold term (i.e., $X_{1 i j} I\left(X_{3 i j}<0.3\right)$ ), and an interaction term between an individuallevel confounder and a cluster-level confounder (i.e., $X_{2 i j} W_{j}$ ).

https://github.com/youmisuk/robustCF 
4. Generate the potential outcomes $Y_{i j}(1), Y_{i j}(0)$ and observed outcome $Y_{i j}$ from the regression model below.

$$
\begin{aligned}
& Y_{i j}(z)=70+2 X_{1 i j}+2 X_{2 i j}+2 X_{3 i j}+2 W_{j}+2 X_{1 i j}^{2}+2 X_{2 i j} W_{j}+2 X_{1 i j} I\left(X_{3 i j}<0.3\right)+ \\
& \beta_{1} U_{j}+z\left(2+2 X_{3 i j}+2 W_{j}+\beta_{2} U_{j}\right)+r_{i j} \\
& Y_{i j}=Z_{i j} Y_{i j}(1)+\left(1-Z_{i j}\right) Y_{i j}(0), \quad r_{i j} \sim N(0,1)
\end{aligned}
$$

Here, $r_{i j}$ is the random error for individual $i$ in cluster $j$. The term $\beta_{1}$ is the main effect of $U_{j}$ on the outcome and the term $\beta_{2}$ is the cross-level interaction effect between $U_{j}$ and the treatment variable. The population ATE $\tau$ is $E\left(2+2 X_{3 i j}+2 W_{j}+\beta_{2} U_{j}\right)=3$. We used $\beta_{1} \in\{2,4\}$ and $\beta_{2} \in\{0,2,4\}$.

Figure 1 summarizes the percent bias of the ATE estimates across different sample size conditions and magnitudes of $\beta_{1}, \beta_{2}$. Note that omitting means for conditions that exceeded $50 \%$ bias permitted clearer comparison of the less biased estimators; Appendix B contains the exact numerical results. The $\mathrm{x}$-axis within each subplot varies the magnitude of the cross-level interaction effect term $\beta_{2}$. Each row of Figure 1 varies the magnitude of the main effect term $\beta_{1}$ and each column represents different configurations of the number of clusters (the first value in the parenthesis) and the mean cluster size (the second value in the parenthesis).

Among non-ML methods, the marginal IPW estimator with propensity scores from random effects logistic regression (Marginal+RePS) had the largest bias across all the conditions. However, as expected, these biases were significantly attenuated when we used clustered weights (Clustered+RePS) instead of marginal weights since the former aggregates cluster-specific ATE estimates that are more robust to biases arising from unmeasured cluster-level confounders.

The percent bias of the marginal estimator with fixed effects logistic regression (Marignal+FePS) generally agreed with that of the clustered estimator with fixed effects propensity scores (Clustered+FePS); they produced nearly unbiased estimates in the absence of cross-level interaction (i.e., $\beta_{2}=0$ ) and small biases in the presence of a cross-level interaction (i.e., $\left.\beta_{2} \neq 0\right)$. More generally, the marginal IPW estimator with fixed effects logistic regression (Marginal+FePS), the clustered IPW estimators, and the DRCGEE estimator were all affected by an increase in $\beta_{2}$, but not in $\beta_{1}$. This result is expected given that these estimators can control an unobserved cluster-level effect either by using cluster weights or fixed effects. In contrast, the marginal IPW estimator with random effects logistic regression (Marginal+RePS) became biased when either $\beta_{1}$ or $\beta_{2}$ increased. Finally, as predicted from Zetterqvist et al. (2016), the DRCGEE estimator performed the best when there was no cross-level interaction effect. However, when a cross-level interaction was present, the DRCGEE estimator produced more biased estimates, especially compared to those from marginal IPW estimators with fixed effects propensity scores (Marginal+FePS) and the clustered IPW estimator with fixed effects propensity scores (Clustered+FePS).

Across different sample size conditions, when the mean cluster size was the smallest (i.e., $(400,10))$, the estimates from the clustered IPW estimators became less accurate. Also, under this condition, the estimates with fixed effects propensity scores were more biased because fixed effects models had 400 cluster indicator variables. When the mean cluster size increased from $(400,10)$ to $(20,200)$, we saw that the marginal estimator with random effects propensity scores (Marginal+RePS) improved and its performance was closer to the marginal estimator with fixed effects logistic regression (Marginal+FePS). This is expected since random effects models converge to fixed effects models as the mean cluster size increases.

Among ML methods, all of our modifications except for the modification based on cluster dummy variables led to smaller bias compared to using the default tuning procedures for Causal Forests $(\mathrm{CF})$. In particular, our third modification based on cluster dummy variables $(\mathrm{CF}+$ ClusterID $)$ performed the worst, producing more than $100 \%$ relative bias in a few con- 


\section{Non-Machine-Learning Methods}

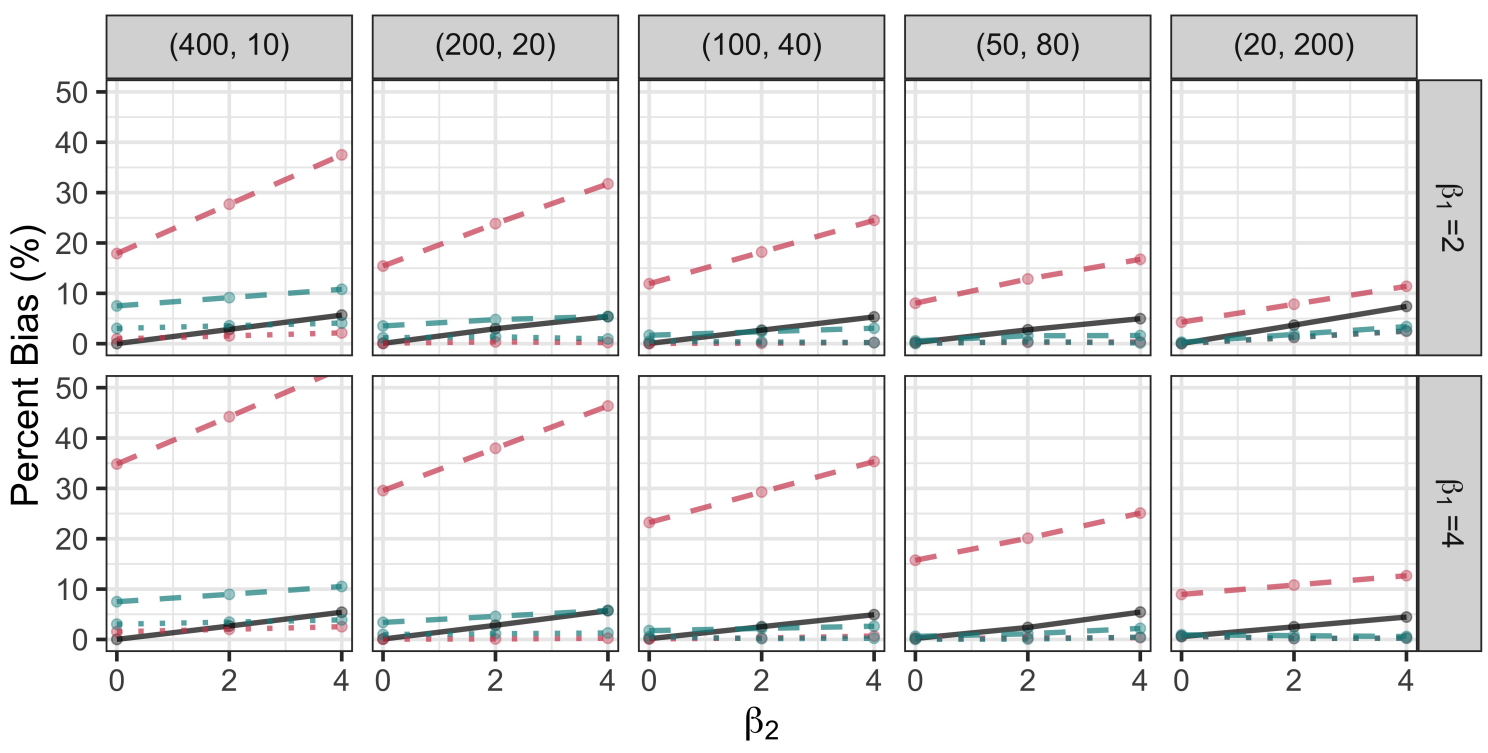

Estimator - DRCGEE $\rightarrow$ Marginal+RePS $: \circ$ Marginal+FePS $\Rightarrow$ Clustered+RePS $=$ : Clustered+FePS

\section{Machine Learning Methods}
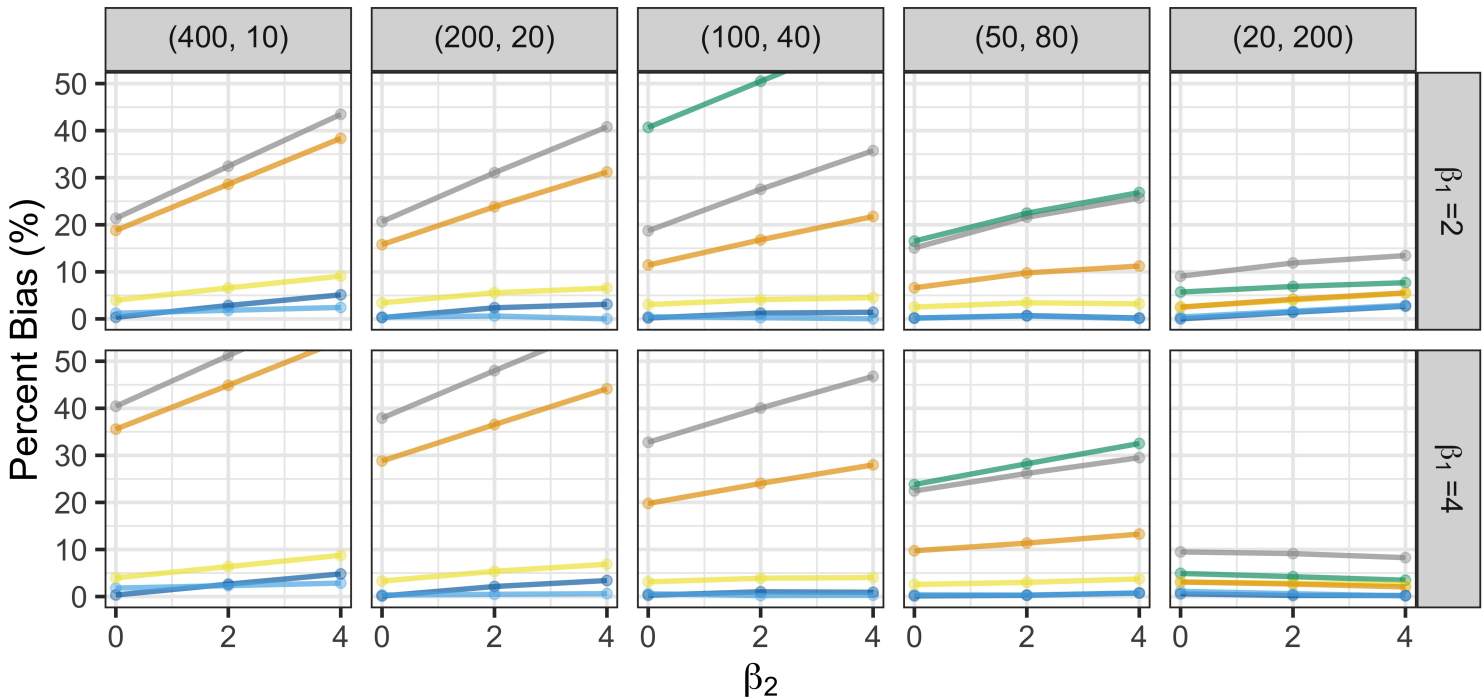

$$
\begin{aligned}
& \text { Estimator }=\mathrm{CF}=\mathrm{CF}+\mathrm{RePS}=\mathrm{CF}+\mathrm{FePS}=\mathrm{CF}+\text { Demean } \\
& -\mathrm{CF}+\text { Demean+PS }
\end{aligned}
$$

Figure 1: Performance of average treatment effect estimates in Design 1. The terms $\beta_{1}$ and $\beta_{2}$ represent the main effect term and the cross-level interaction effect term in the outcome model. Two values in the parentheses represent the number of clusters and the mean cluster size, respectively. All conditions for which percent bias exceeded $50 \%$ were omitted to permit clearer comparison of the less biased estimators. DRCGEE $=$ the doubly robust conditional generalized estimating equation estimator; Marginal $=$ the marginal inverse propensity weighting estimator; Clustered $=$ the clustered inverse propensity weighting estimator; RePS = propensity scores from random effects logistic regression; FePS = propensity scores from fixed effects logistic regression; $\mathrm{CF}=$ Causal Forests without any modifications; $\mathrm{CF}+\mathrm{RePS}=\mathrm{CF}$ with propensity scores from random effects logistic regression; $\mathrm{CF}+\mathrm{FePS}=\mathrm{CF}$ with propensity scores from fixed effects logistic regression; $\mathrm{CF}+\mathrm{ClusterID}=\mathrm{CF}$ with the additional set of cluster dummies; $\mathrm{CF}+$ Demean $=\mathrm{CF}$ with demeaned observed variables; $\mathrm{CF}+$ Demean $+\mathrm{PS}=\mathrm{CF}$ with demeaned observed variables and propensity scores trained with demeaned variables. 
ditions (not shown in Figure 1). However, the performance of $\mathrm{CF}+\mathrm{ClusterID}$ improved dramatically when the mean cluster size increased from $(400,10)$ to $(20,200)$. The second and fifth modifications based on using fixed effects propensity scores or demeaned variables with propensity scores $(\mathrm{CF}+\mathrm{FePS}, \mathrm{CF}+$ Demean $+\mathrm{PS})$ had the smallest bias, followed by the fourth modification based on using demeaned variables $(\mathrm{CF}+$ Demean $)$. The first modification based on random effects propensity scores $(\mathrm{CF}+\mathrm{RePS})$ performed somewhere between $\mathrm{CF}+\mathrm{ClusterID}$ and the other modifications; its performance improved as the mean cluster size increased. We saw that the performances of $\mathrm{CF}+\mathrm{FePS}, \mathrm{CF}+$ Demean, and $\mathrm{CF}+$ Demean $+\mathrm{PS}$ remained relatively stable across different sample size conditions. Across different values of $\beta_{1}$ and $\beta_{2}, \mathrm{CF}$, $\mathrm{CF}+\mathrm{RePS}$, and $\mathrm{CF}+\mathrm{ClusterID}$ produced more bias when either $\beta_{1}$ or $\beta_{2}$ increased. In contrast, $\mathrm{CF}+\mathrm{FePS}, \mathrm{CF}+$ Demean, and $\mathrm{CF}+$ Demean $+\mathrm{PS}$ produced no bias when $\beta_{1}$ increased, but produced some bias when $\beta_{2}$ increased. Interestingly, the bias arising from varying $\beta_{1}$ and $\beta_{2}$ noticeably decreased when the mean cluster size was larger than the number of clusters, i.e., $(20,200)$.

Comparing non-ML methods and ML methods, if $\beta_{1} \neq 0$ but $\beta_{2}=0$, the performance of the modified Causal Forests with fixed effects or demeaned propensity scores $(\mathrm{CF}+\mathrm{FePS}$, $\mathrm{CF}+$ Demean+PS) was competitive to that of the DRCGEE estimator and the IPW-based estimators using propensity scores from fixed effects logistic regression (Marginal+FePS, Clustered + FePS). When both $\beta_{1} \neq 0$ and $\beta_{2} \neq 0$, the modified Causal Forests with fixed effects or demeaned propensity scores was as robust as the IPW-based estimators using propensity scores from fixed effects logistic regression and performed better than the DRCGEE estimator. We remark that we observed similar performance patterns with respect to RMSE (see Appendix B for details).

\subsection{Design 2: Uniformly Distributed $U_{j}$ and Linear Outcome Model}

Design 2 used the same data generating models as Design 1 except that the unmeasured clusterlevel confounder $U_{j}$ was uniformly distributed, i.e., $U_{j} \sim \operatorname{Unif}(-2,2)$. We summarize the percent bias of the ATE estimates in Figure 2, Similar to Figure 1, Figure 2 only shows percent bias ranging from $0 \%$ to $50 \%$ and Appendix C contains the exact numerical results. As expected, the marginal IPW estimator with random effects propensity scores (Marginal+RePS) and the modified Causal Forests with random effects propensity scores (CF+RePS) performed worse under Design 2 than Design 1 because we violate the underlying distributional assumption of random effects models. The additional bias from $\mathrm{CF}+\mathrm{RePS}$ was similar to that from Marginal+RePS when the cluster sizes were small (i.e., $(400,10),(200,20))$. But when the cluster sizes increased, the additional bias from $\mathrm{CF}+\mathrm{RePS}$ was smaller than that from Marginal+RePS. We also saw that under non-Normally distributed $U_{j}$, the default Causal Forests $(\mathrm{CF})$ and the modified Causal Forests with cluster dummy variables $(\mathrm{CF}+$ ClusterID) were more biased unless the cluster sizes were very large. Finally, Marginal+RePS, CF, CF+RePS, and CF+ClusterID produced more bias when either $\beta_{1}$ or $\beta_{2}$ increased and all of them generally had larger biases under Design 2 than Design 1.

On the other hand, the performance of the clustered IPW estimator with random effects propensity scores (Clustered+RePS) remained almost the same because the clustered estimator mitigates biases arising from unmeasured cluster-level confounders through clustered weights. Also, the estimators based on fixed effects or demeaning approaches show robust performance because they did not make any assumptions on the distribution of $U_{j}$. Specifically, the modified Causal Forests based on fixed effects propensity scores or demeaned variables ( $\mathrm{CF}+\mathrm{FePS}$, $\mathrm{CF}+$ Demean, $\mathrm{CF}+$ Demean $+\mathrm{PS})$, the marginal or clustered estimators with fixed effects logistic regression (Marginal+FePS, Clustered+FePS), and the DRCGEE estimator produced very 


\section{Non-Machine-Learning Methods}
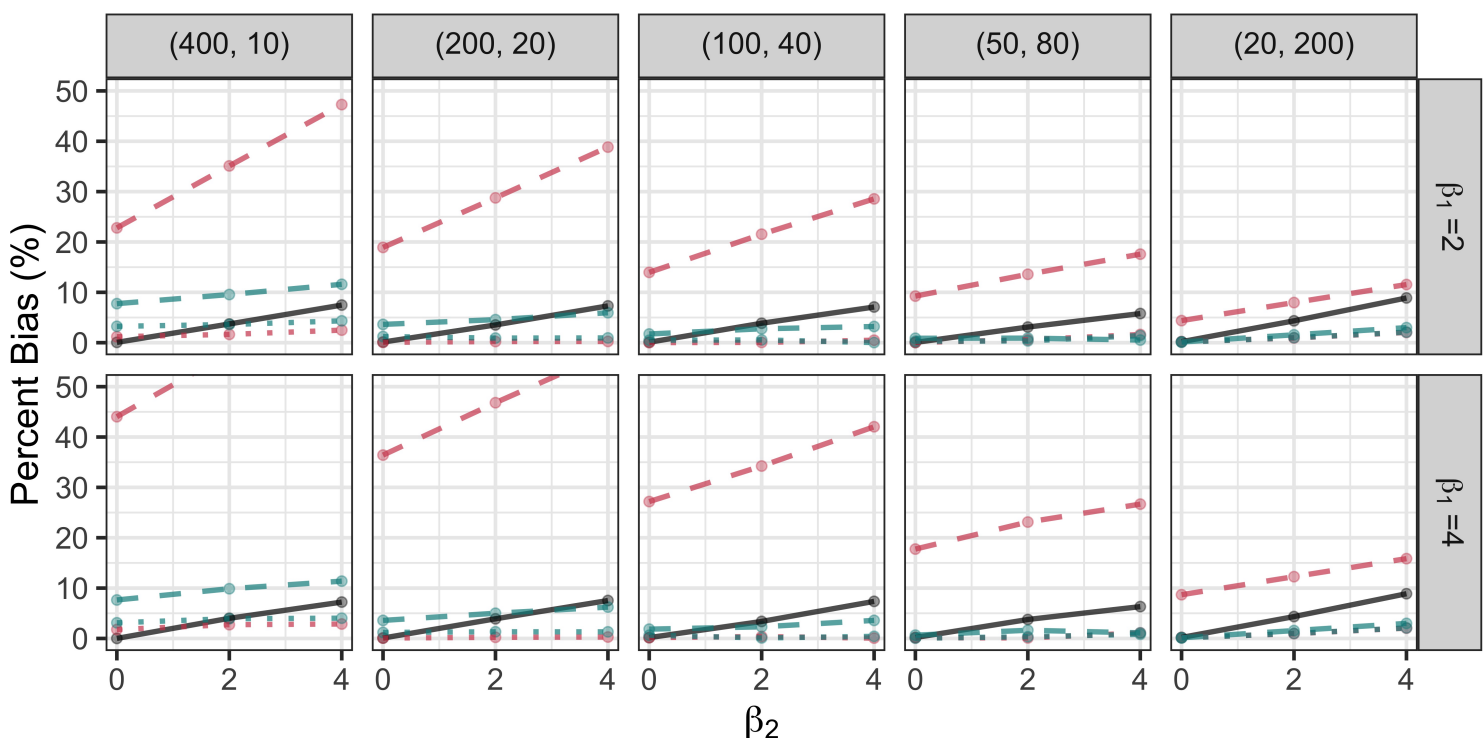

Estimator - DRCGEE $\rightarrow$ Marginal+RePS $: \circ$ Marginal+FePS - Clustered+RePS $=$ : Clustered+FePS

\section{Machine Learning Methods}
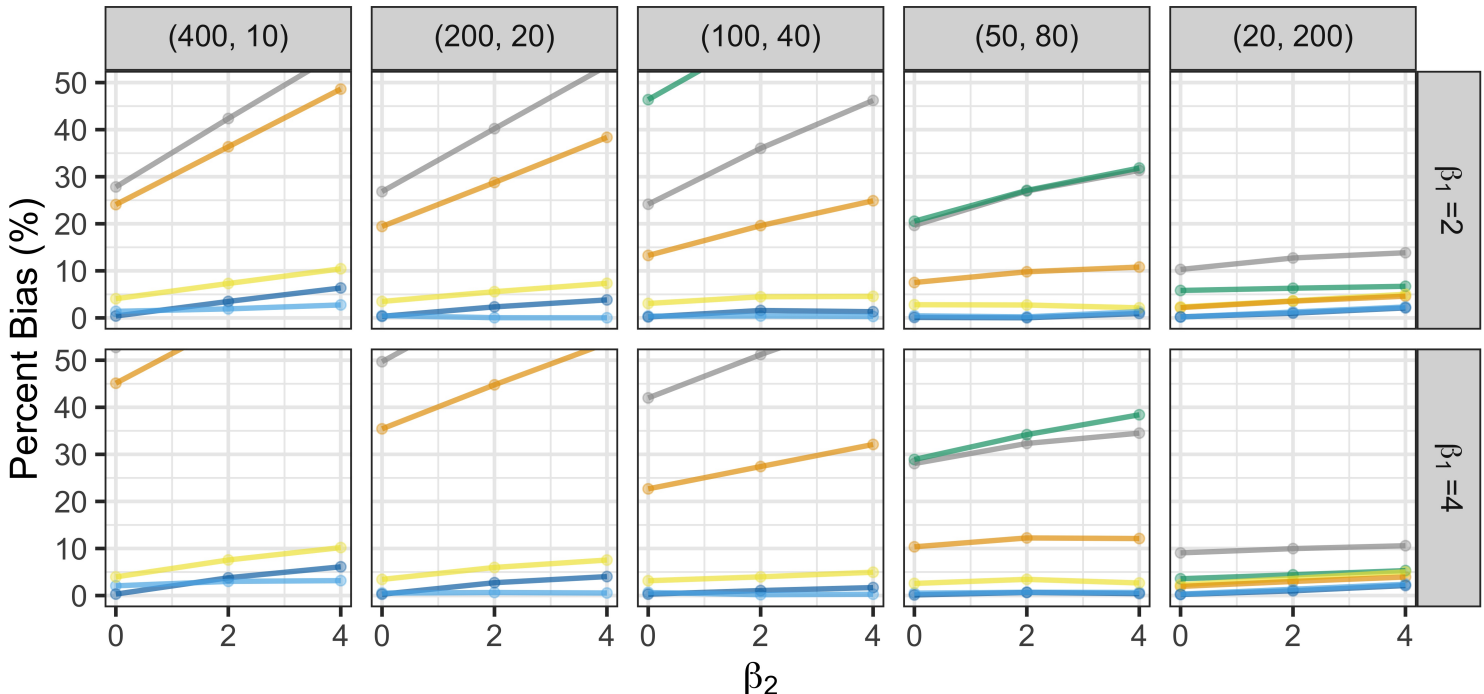

$$
\begin{aligned}
\text { Estimator } & -\mathrm{CF}=\mathrm{CF}+\mathrm{RePS}=\mathrm{CF}+\mathrm{FePS}=\mathrm{CF}+\text { ClusterID }-\mathrm{CF}+\text { Demean } \\
& -\mathrm{CF}+\text { Demean+PS }
\end{aligned}
$$

Figure 2: Performance of average treatment effect estimates in Design 2. The terms $\beta_{1}$ and $\beta_{2}$ represent the main effect term and the cross-level interaction effect term in the outcome model. Two values in the parentheses represent the number of clusters and the mean cluster size, respectively. All conditions for which percent bias exceeded $50 \%$ were omitted to permit clearer comparison of the less biased estimators. DRCGEE $=$ the doubly robust conditional generalized estimating equation estimator; Marginal $=$ the marginal inverse propensity weighting estimator; Clustered $=$ the clustered inverse propensity weighting estimator; RePS = propensity scores from random effects logistic regression; FePS = propensity scores from fixed effects logistic regression; $\mathrm{CF}=$ Causal Forests without any modifications; $\mathrm{CF}+\mathrm{RePS}=\mathrm{CF}$ with propensity scores from random effects logistic regression; $\mathrm{CF}+\mathrm{FePS}=\mathrm{CF}$ with propensity scores from fixed effects logistic regression; $\mathrm{CF}+\mathrm{ClusterID}=\mathrm{CF}$ with the additional set of cluster dummies; $\mathrm{CF}+$ Demean $=\mathrm{CF}$ with demeaned observed variables; $\mathrm{CF}+$ Demean $+\mathrm{PS}=\mathrm{CF}$ with demeaned observed variables and propensity scores trained with demeaned variables. 
similar estimates as those from Design 1; the differences in percent bias were generally less than 1\%. Also, similar to Design 1, the estimators based on fixed effects or demanding approaches yielded no bias when $\beta_{1}$ increased, but produced small bias when $\beta_{2}$ increased. We observed similar results in terms of RMSE (see Appendix C for details).

\subsection{Design 3: Uniformly Distributed $U_{j}$, Linear Outcome Model, and Misspecified Working Models}

In this simulation, we used the same design as Design 2, but we ignored higher-order terms when fitting the outcome or the propensity score model. Note that we also misspecified the propensity scores going into our modified Causal Forests. This design reflects a more realistic situation in which a careful investigator, despite his/her best attempts, incorrectly specified the two models. Figure 3 summarizes the percent bias of the ATE estimates. Similar to Figures 1 and 2, Figure 33s y-axis only shows percent bias ranging from $0 \%$ to $50 \%$ and Appendix $\mathrm{D}$ contains the exact numerical results. Not surprisingly, among non-ML methods, all the estimators except for the DRCGEE estimator with the mis-specified propensity score model but a correctly specified outcome model (DRCGEE+PS) produced more biased estimates compared to the corresponding estimators in Design 2; the estimates from Design 3 were at least $9 \%$ more biased across all simulation conditions than those from Design 2. DRCGEE+PS produced almost the same estimates as the DRCGEE estimator in Design 2 because of the doubly robust property of the DRCGEE estimator. But when we mis-specified both the propensity score model and the outcome model in the DRCGEE estimator (DRCGEE+both), the estimates were as biased as those from the clustered IPW estimators.

Similar to Designs 1 and 2, the magnitude of the biases depended on the underlying magnitudes of unmeasured cluster-level confounding. Specifically, the marginal IPW estimator with random effects logistic regression (Marginal+RePS) became biased when either $\beta_{1}$ or $\beta_{2}$ increased. The bias in Marginal+RePS could be reduced by implementing the clustered IPW estimator (Clustered+RePS). In contrast, the marginal IPW estimator with fixed effects logistic regression (Marginal+FePS), the clustered IPW estimators, and the DRCGEE estimator were affected by $\beta_{2}$, but not $\beta_{1}$.

Among ML methods, injecting misspecified propensity scores into Causal Forests diminished the effectiveness of our modifications and produced more biased estimates than the corresponding modified Causal Forests in Design 2. Nevertheless, CF+FePS produced the smallest bias followed by $\mathrm{CF}+$ Demean+PS; the performance of $\mathrm{CF}+\mathrm{FePS}$ was closer to that of the modified Causal Forests with demeaned variables $(\mathrm{CF}+$ Demean $)$ in Design 2. Also, similar to Designs 1 and 2 , across different values of $\beta_{1}$ and $\beta_{2}, \mathrm{CF}+\mathrm{RePS}$ produced more bias when either $\beta_{1}$ or $\beta_{2}$ increased, whereas $\mathrm{CF}+\mathrm{FePS}$ and $\mathrm{CF}+$ Demean $+\mathrm{PS}$ were affected by $\beta_{2}$, not $\beta_{1}$. Most importantly, $\mathrm{CF}+\mathrm{FePS}$ and $\mathrm{CF}+$ Demean+PS were less biased than all non-ML methods with misspecified models. That is, the impact of misspecifying propensity scores in Causal Forests was not as great as misspecifying them in non-ML methods. Similar results were obtained with respect to RMSE (see Appendix $\mathrm{D}$ for details). 


\section{Non-Machine-Learning Methods}
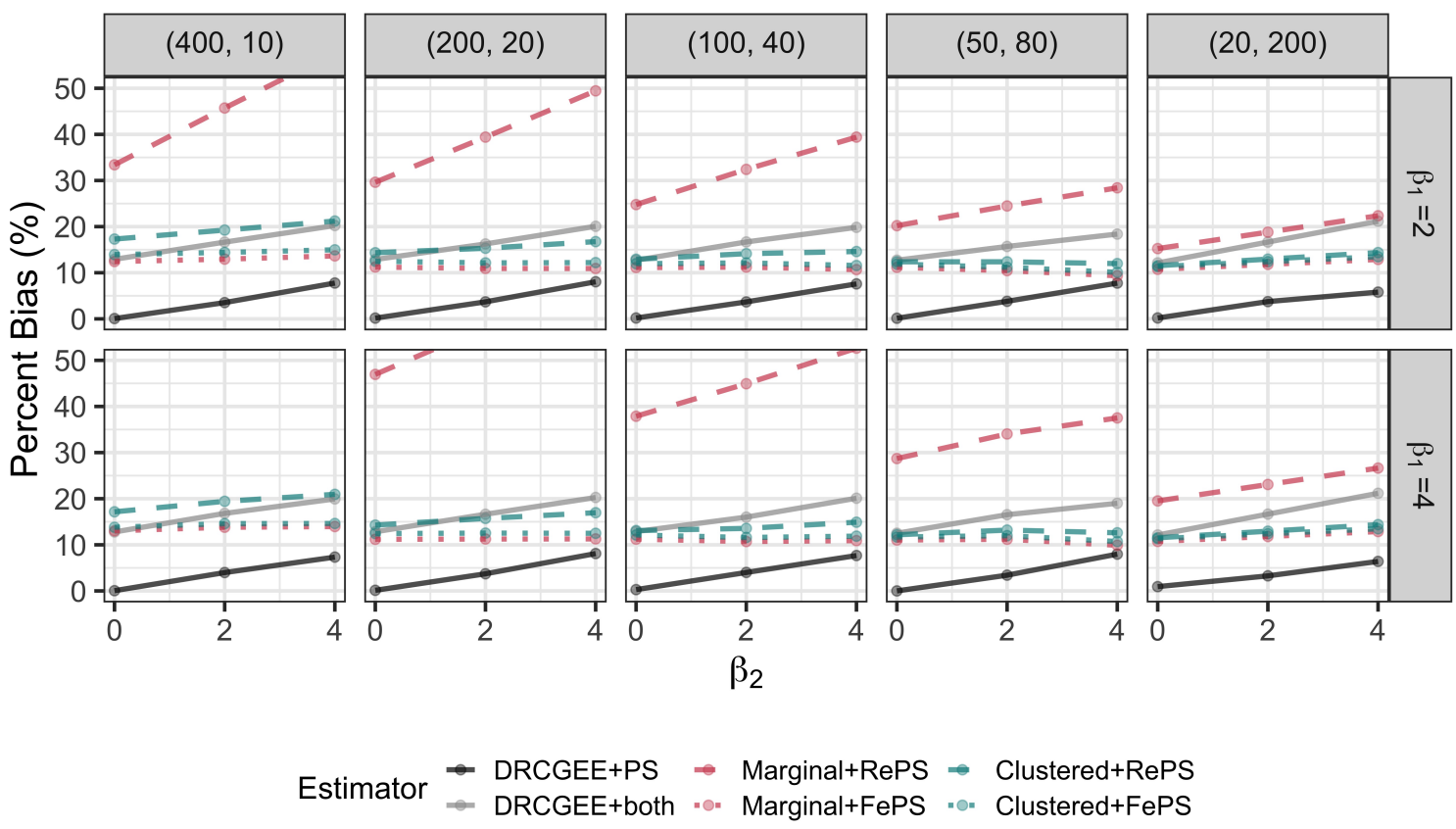

\section{Machine Learning Methods}
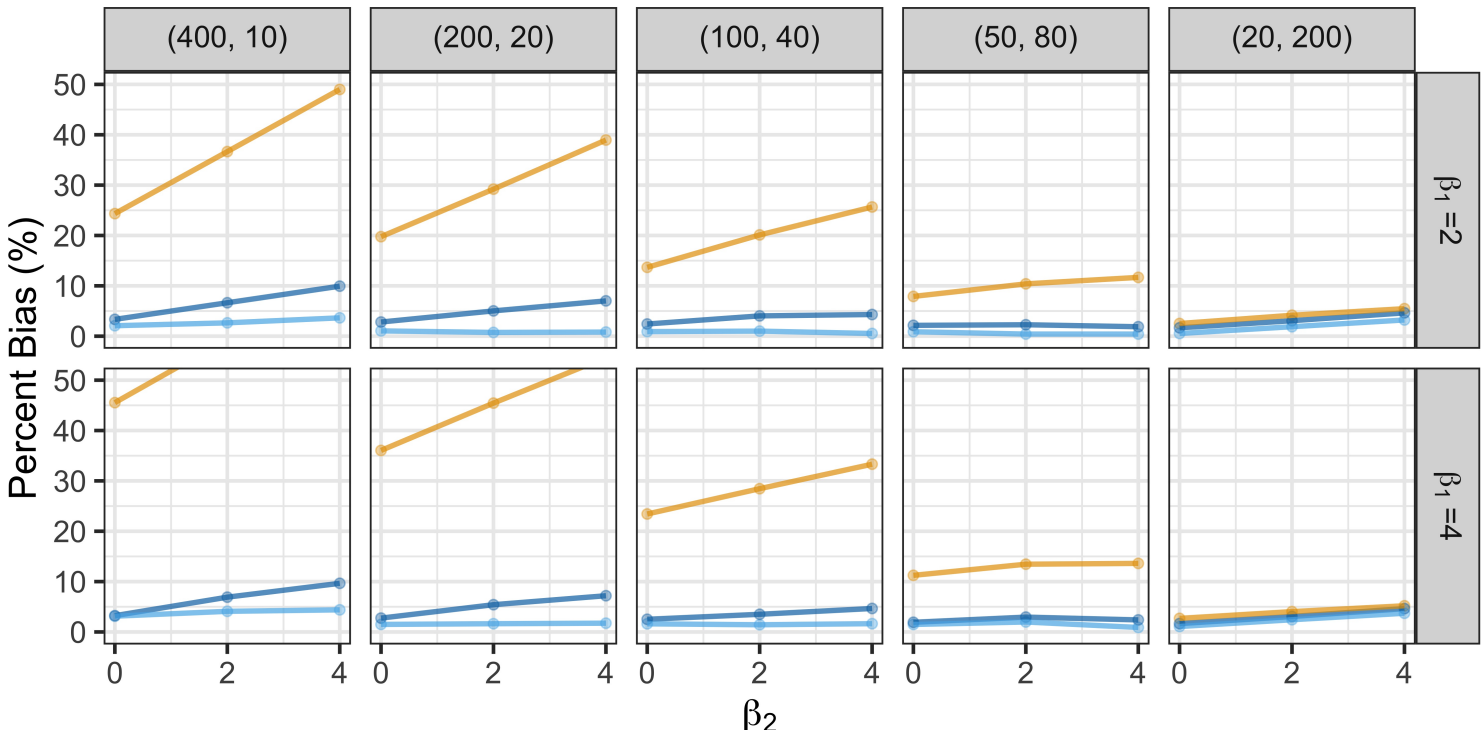

Estimator $-\mathrm{CF}+\mathrm{RePS}-\mathrm{CF}+\mathrm{FePS}-\mathrm{CF}+$ Demean+PS

Figure 3: Performance of average treatment effect estimates in Design 3. The terms $\beta_{1}$ and $\beta_{2}$ represent the main effect term and the cross-level interaction effect term in the outcome model. Two values in the parentheses represent the number of clusters and the mean cluster size, respectively. All conditions for which percent bias exceeded $50 \%$ were omitted to permit clearer comparison of the less biased estimators. DRCGEE+PS = the doubly robust conditional generalized estimating equation estimator with mis-specified propensity scores; DRCGEE + both $=$ the doubly robust conditional generalized estimating equation estimator with mis-specified propensity scores and mis-specified outcome model; Marginal = the marginal inverse propensity weighting estimator; Clustered $=$ the clustered inverse propensity weighting estimator; RePS = propensity scores from mis-specified random effects logistic regression; FePS = propensity scores from mis-specified fixed effects logistic regression; $\mathrm{CF}+\mathrm{RePS}=\mathrm{CF}$ with propensity scores from mis-specified random effects logistic regression; $\mathrm{CF}+\mathrm{FePS}=\mathrm{CF}$ with propensity scores from mis-specified fixed effects logistic regression; $\mathrm{CF}+$ Demean $+\mathrm{PS}=\mathrm{CF}$ with demeaned observed variables and mis-specified propensity scores trained with demeaned variables. 


\subsection{Design 4: Uniformly Distributed $U_{j}$ and Nonlinear Binary Outcome Model}

Our last design followed Design 2, except we used a nonlinear, binary outcome model rather than a linear outcome model. Specifically, we changed the outcome model to:

$$
\begin{aligned}
& \operatorname{logit}\left(P\left(Y_{i j}(z)=1 \mid \mathbf{X}_{i j}, W_{j}, U_{j}\right)\right) \\
&=-0.6+0.3 X_{1 i j}+0.3 X_{2 i j}+0.3 X_{3 i j}+0.3 W_{j}+0.3 X_{1 i j}^{2}+0.3 X_{2 i j} W_{j} \\
&+0.3 X_{1 i j} I\left(X_{3 i j}<0.3\right)+0.3 U_{j}+\beta_{1} U_{j}+z\left(0.5+0.3 X_{3 i j}+0.3 W_{j}+\beta_{2} U_{j}\right) \\
& Y_{i j}(z) \sim \operatorname{Bernoulli}\left(P\left(Y_{i j}(z)=1 \mid \mathbf{X}_{i j}, W_{j}, U_{j}\right)\right), \quad Y_{i j}=Z_{i j} Y_{i j}(1)+\left(1-Z_{i j}\right) Y_{i j}(0)
\end{aligned}
$$

We used $\beta_{1} \in\{0.3,0.9\}$ and $\beta_{2} \in\{0,0.3,0.9\}$. Our target estimand is still the ATE and because of the nonlinearity of the outcome model, we used Monte Carlo integration with 1,000,000 replications to compute the population ATE $\tau$.

Figure 4 summarizes the percent bias of the ATE estimates in Design 4. The figure only shows percent bias from $0 \%$ to $3 \%$ bias; the numerical results in Appendix E report all the percent biases. Generally, the relative performance between the estimators was similar to previous designs under a linear outcome model. Among non-ML methods, we observed that the marginal estimator with random effects propensity scores (Marginal+RePS) showed the worst performance. When we used random effects propensity scores with the clustered estimator, biases decreased across all the conditions. In contrast, the marginal or clustered estimator with fixed effects propensity scores (Marginal+FePS, Clustered+FePS) generally showed the best performance across all the conditions. This behavior is not surprising especially since IPW-based methods do not rely on the outcome model.

Among ML methods, similar to Designs 1 and 2, all modifications except for the modification with cluster indicators (CF+ClusterID) reduced bias compared to the default Causal Forests (CF). Causal Forests with fixed effects or demeaned propensity scores (CF+FePS, $\mathrm{CF}+$ Demean+PS) were the top two performers, followed by Causal Forests using demeaned variables $(\mathrm{CF}+$ Demean). Including random effects propensity scores in Causal Forests (CF+RePS) was less effective in reducing biases from unmeasured cluster-level confounders.

Between non-ML methods and ML methods, when the cross-level interaction was absent (i.e., $\left.\beta_{1} \neq 0, \beta_{2}=0\right)$, the performance of the modified Causal Forests with fixed effects or demeaned propensity scores $(\mathrm{CF}+\mathrm{FePS}, \mathrm{CF}+$ Demean $+\mathrm{PS})$ was comparable to that of the DRCGEE estimator, Marginal+FePS, and Clustered+FePS. When the cross-level interaction was present (i.e., $\beta_{1} \neq 0, \beta_{2} \neq 0$ ), the modified Causal Forests with fixed effects propensity scores was as robust as the IPW-based estimators using fixed effects propensity scores and outperformed the DRCGEE estimator. Similar performance patterns were observed with respect to RMSE (see Appendix E for details).

\subsection{Takeaways from Simulation Studies}

Overall, across the different simulation designs, two of our proposed ML modifications, $\mathrm{CF}+\mathrm{FePS}$ and $\mathrm{CF}+$ Demean $+\mathrm{PS}$, were the only methods that remained robust. $\mathrm{CF}+\mathrm{FePS}$ and $\mathrm{CF}+$ Demean +PS had either the lowest or one of the lowest percent biases in each simulation design. Both $\mathrm{CF}+\mathrm{FePS}$ and $\mathrm{CF}+$ Demean+PS performed better than the default Causal Forests without any modifications, demonstrating that our simple modifications to the ML method worked better than using the default tuning procedures. When we used correctly specified propensity scores, the two top performers were competitive to the IPW-based estimators using propensity scores from fixed effects logistic regression. But when the working propensity score model inside the modified Causal Forests was misspecified, the modified Causal Forests produced more robust estimates of the ATE than the IPW estimators using the same mis-specified propensity score. 
Non-Machine-Learning Methods

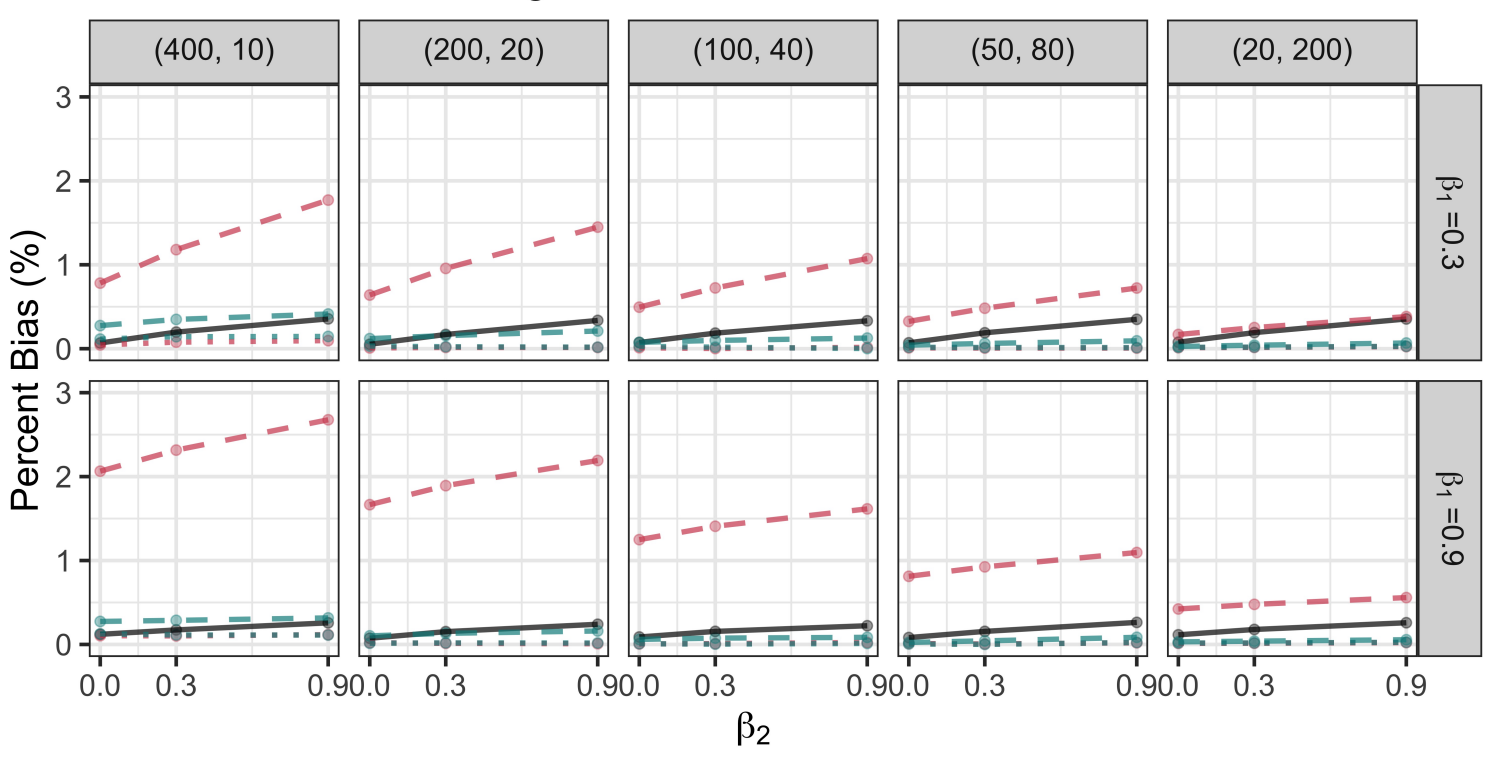

Estimator - DRCGEE $\Rightarrow$ Marginal+RePS $=$ : Marginal+FePS $\Rightarrow$ Clustered+RePS $: \circ$ Clustered+FePS

Machine Learning Methods

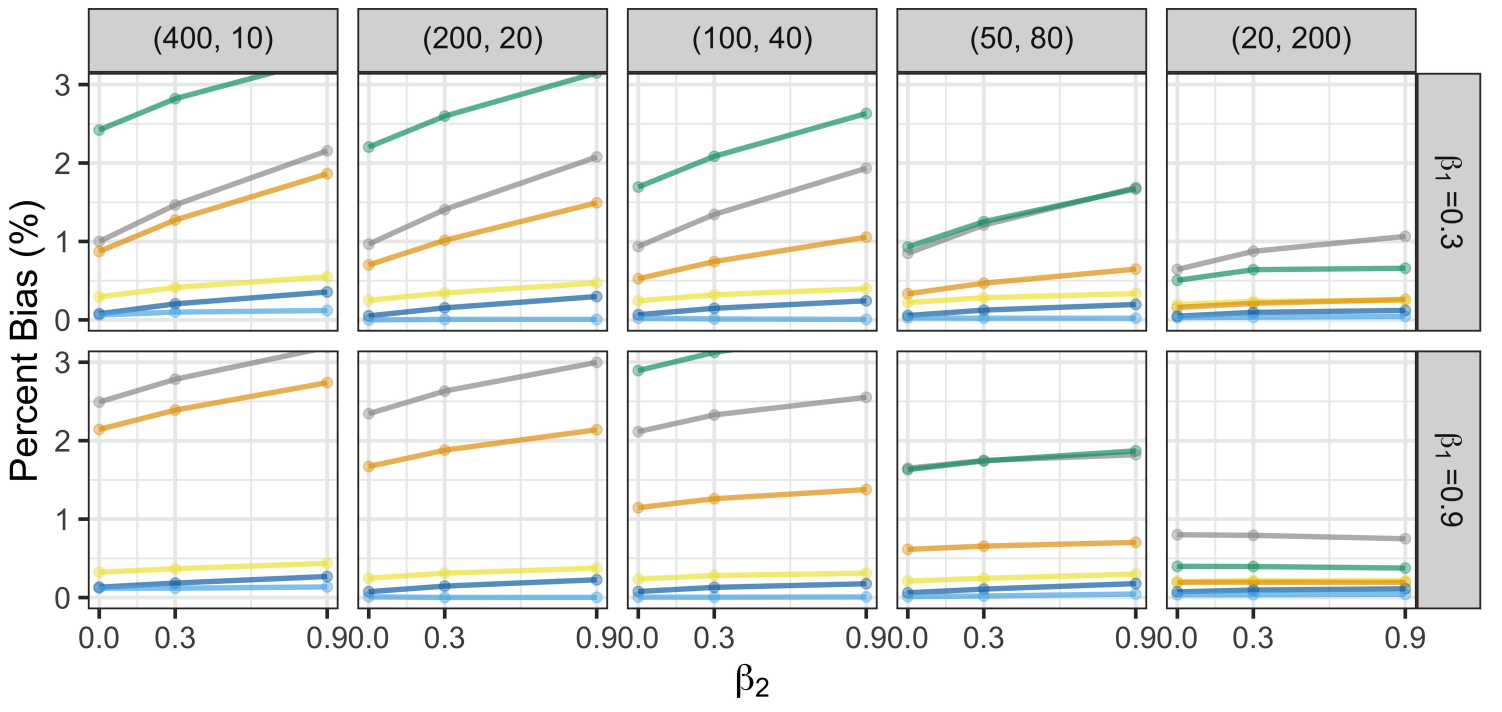

$\begin{aligned} & \text { Estimator }-\mathrm{CF}=\mathrm{CF}+\mathrm{RePS}-\mathrm{CF}+\mathrm{FePS}-\mathrm{CF}+\text { ClusterlD }-\mathrm{CF}+\text { Demean } \\ &-\mathrm{CF}+\text { Demean+PS }\end{aligned}$

Figure 4: Performance of average treatment effect estimates in Design 4. The terms $\beta_{1}$ and $\beta_{2}$ represent the main effect term and the cross-level interaction effect term in the outcome model. Two values in the parentheses represent the number of clusters and the mean cluster size, respectively. All conditions for which percent bias exceeded $50 \%$ were omitted to permit clearer comparison of the less biased estimators. DRCGEE $=$ the doubly robust conditional generalized estimating equation estimator; Marginal $=$ the marginal inverse propensity weighting estimator; Clustered $=$ the clustered inverse propensity weighting estimator; RePS = propensity scores from random effects logistic regression; FePS $=$ propensity scores from fixed effects logistic regression; $\mathrm{CF}=$ Causal Forests without any modifications; $\mathrm{CF}+\mathrm{RePS}=\mathrm{CF}$ with propensity scores from random effects logistic regression; $\mathrm{CF}+\mathrm{FePS}=\mathrm{CF}$ with propensity scores from fixed effects logistic regression; $\mathrm{CF}+\mathrm{ClusterID}=\mathrm{CF}$ with the additional set of cluster dummies; $\mathrm{CF}+$ Demean $=\mathrm{CF}$ with demeaned observed variables; $\mathrm{CF}+$ Demean $+\mathrm{PS}=\mathrm{CF}$ with demeaned observed variables and propensity scores trained with demeaned variables. 
Based on these observations, we would recommend that investigators use either $\mathrm{CF}+\mathrm{FePS}$ or $\mathrm{CF}+$ Demean+PS when they are working with multilevel observational studies and there is a concern for unmeasured cluster-level confounding.

Our simulation studies also revealed that $\mathrm{CF}+\mathrm{RePS}$ and $\mathrm{CF}+\mathrm{ClusterID}$ are less robust and more sensitive to the underlying data generating process. For example, when the number of clusters was larger than the mean cluster size, $\mathrm{CF}+$ ClusterID had relative bias that exceeded $100 \%$. But when the number of clusters was smaller than the mean cluster size, CF+ClusterID had much smaller bias. When the distribution of the cluster-level unmeasured confounder $U_{j}$ was non-Normal, CF+RePS produced some bias. But this bias was reduced when the number of clusters was smaller than the mean cluster size. Also, when $U_{j}$ was Normally distributed, $\mathrm{CF}+\mathrm{RePS}$ had lower bias compared to when $U_{j}$ was non-Normally distributed. Given the sensitivity of these two modifications to the underlying data generating process, we do not recommend that investigators use them in practice.

Finally, as with all simulation studies, our simulation studies do not encompass every possible data generating model for multilevel observational studies and hence, some of our advice may fail to hold in other data generating models. However, given the diversity of data generating models that we have considered thus far (e.g., linear/non-linear outcomes, non-Normal unmeasured cluster-level confounders, higher-order or threshold terms) and the robust performance of $\mathrm{CF}+\mathrm{FePS}$ and $\mathrm{CF}+$ Demean $+\mathrm{PS}$ across all the data generating models, we are cautiously optimistic that they will perform well in other multilevel observational studies. At the very least, we believe that investigators can use them as preliminary, baseline non-parametric estimators of the ATE and make additional adjustments based on the data at hand.

\section{Real Data Study}

\subsection{Data and Variables}

We illustrate our modifications of Causal Forests by investigating the effects of taking an eighthgrade algebra course from the ECLS-K data. ECLS-K is a longitudinal study sponsored by the National Center for Education Statistics (NCES) to study school performance and student experience using a dual-frame multistage sampling design. The data were collected from the fall of 1998 to the spring of 2017 (Walston \& McCarroll, 2010); the ECLS-K website http:// nces.ed.gov/ecls/kindergarten.asp contains more information about the ECLS-K data collection process. Our analysis used data from the fifth-grade assessments in the spring of 2004 and the eighth-grade assessments in the spring of 2007. We used the fifth-grade assessment data for pre-treatment covariates and the eighth-grade assessments data for the treatment and the outcome.

Specifically, the treatment variable is whether a student took an eighth-grade algebra course in the spring of 2007, with 1 indicating that the student took an algebra or more advanced course and 0 otherwise. The outcome was students' math achievement scores in the spring of 2007. According to the ECLS-K study report, student assessments (i.e., the outcome variable) for 8th graders were conducted at least two months after the beginning of the Spring semester (Tourangeau et al., 2009); the students often started their year-long algebra course in the Fall semester. Based on this information, we assume that the outcome was measured after the treatment was delivered. We included 11 pre-treatment covariates that were thought to influence both the treatment and the outcome based on prior research (Rickles, 2013 Walston \& McCarroll, 2010). Eight of these eleven covariates were student-level covariates and included prior math achievement scores, parents' expectation of their child's highest level of education, gender, race, socio-economic status, poverty level, mother's educational level, and family type (living with one parent or two parents). The rest of the covariates were school-level covariates 
and included public or private school, school location (urban, suburb, small town), and region (West, Midwest, Northeast, South). All continuous covariates were standardized to have a mean of 0 and a standard deviation of 1 . The analytic sample consisted of 2,581 students from 170 schools with a mean cluster size of 15.8 .

We ran Causal Forests with our proposed modifications to estimate the treatment effect on math achievement scores. As a comparison, we also used the same parametric estimators that we used in our simulation studies: the DRCGEE estimator, the marginal IPW estimator with random effects and fixed effects propensity scores, and the clustered IPW estimator with random effects and fixed effects propensity scores. We used the function best_linear_projection in the grf package (Tibshirani et al., 2020) for Causal Forests, the drgee package (Zetterqvist \& Sjölander, 2015) for the DRCGEE estimator, and the Ime4 package (Bates et al., 2015) for random effects logistic regression, all implemented in R. Data and R codes for our real data analysis are available in the supplementary materials (ECLSK_Algebra_complete.csv; DataAnalysisCodes.R) and the first author's GitHub repository]

Based on the cluster size and the number of clusters, our empirical study most closely parallels the $(200,20)$ condition under Designs 2 and 3. Although the true treatment and outcome models for the empirical study are never known, if they are similar to those under Designs 2 and 3, we would expect that our proposed modifications would be able to remove biases arising from omitted cluster-level confounders. Specifically, under these two designs, Causal Forests with fixed effects or demeaned propensity scores $(\mathrm{CF}+\mathrm{FePS}, \mathrm{CF}+\mathrm{Demean}+\mathrm{PS})$ would be expected to produce more accurate and robust estimates.

Lastly, we conducted a sensitivity analysis to assess the sensitivity of our conclusions about the ATE if there were an individual-level unmeasured confounder. Our sensitivity analysis follows a procedure from Hong and Raudenbush (2006) in which we create a hypothetical unmeasured confounder based on the associations between the observed covariates, the treatment, and the outcome, and we report the adjusted confidence interval in the presence of such an unmeasured confounder.

\subsection{Results}

Table 3 provides the ATE estimates of taking the eighth-grade algebra course. As shown in Table 3, the prima facie effect was 13.29 points; this is the unadjusted mean difference in math achievement scores between students who took the algebra or more advanced course and students who did not. The unadjusted estimate was reduced after applying each of the different estimators. The estimate from the DRCGEE estimator was 2.98. The estimates from the marginal or clustered IPW estimators with random effects or fixed effects propensity scores ranged from 1.90 to 4.75 . The estimates with the propensity scores from fixed effects models (Marginal+FePS, Clustered+FePS) were smaller than those with propensity scores from random effects models (Marginal+RePS, Clustered+RePS). Among Causal Forests, the default Causal Forests (CF) yielded an estimate of 2.47. The estimates from modified Causal Forests ranged from 2.54 to 3.22 , except the estimate from the Causal Forests with cluster dummies (CF+ClusterID) which resulted in a relatively high estimate of 4.28. Compared to non-ML methods, Causal Forests with random effects propensity scores $(\mathrm{CF}+\mathrm{RePS})$ produced the same estimate as Causal Forests with fixed effects propensity scores $(\mathrm{CF}+\mathrm{FePS})$. Regarding standard errors, $\mathrm{CF}+\mathrm{FePS}$ produced the largest standard error, whereas Causal Forests using demeaned variables with propensity scores $(\mathrm{CF}+$ Demean $+\mathrm{PS})$ had the smallest standard error. The reduction in standard error may come from both the demeaning procedure and the fitting procedure underlying Causal Forests, especially if the underyling data generating model is non-linear. Overall, all the ATE estimates from ML methods and non-ML methods conclude that there was a positive effect of taking an

https://github.com/youmisuk/robustCF 
eighth-grade algebra course on math achievement scores. This positive effect is consistent with the result of Rickles and Seltzer, 2014 who examined the effect of taking an 8th grade algebra course on math achievement scores, though their sample was from a large urban school district and not based on the ECLS-K data.

Table 3: ATE estimates of taking an eighth-grade algebra course

\begin{tabular}{lcc}
\hline & Estimate & Standard Error \\
\hline Prima facie (unadjusted) & 13.29 & 0.73 \\
DRCGEE & 2.98 & 0.58 \\
Marginal+RePS & 2.83 & 0.75 \\
Marginal+FePS & 1.90 & 0.75 \\
Clustered+RePS & 4.75 & 0.77 \\
Clustered+FePS & 2.72 & 0.92 \\
CF & 2.47 & 0.51 \\
CF+RePS & 2.54 & 0.57 \\
CF+FePS & 2.54 & 0.72 \\
CF+ClusterID & 4.28 & 0.55 \\
CF+Demean & 3.22 & 0.50 \\
CF+Demean+PS & 2.86 & 0.46 \\
\hline
\end{tabular}

Note: Except for the DRCGEE estimator, standard errors were estimated using cluster bootstrap sampling with 5000 replicates. DRCGEE $=$ the doubly robust conditional generalized estimating equation estimator; Marginal $=$ the marginal inverse propensity weighting estimator; Clustered $=$ the clustered inverse propensity weighting estimator; RePS = propensity scores from random effects logistic regression; $\mathrm{FePS}=$ propensity scores from fixed effects logistic regression; $\mathrm{CF}=$ Causal Forests without any modifications; $\mathrm{CF}+\mathrm{RePS}=\mathrm{CF}$ with propensity scores from random effects logistic regression; $\mathrm{CF}+\mathrm{FePS}=\mathrm{CF}$ with propensity scores from fixed effects logistic regression; $\mathrm{CF}+\mathrm{ClusterID}=\mathrm{CF}$ with the additional set of cluster dummies; $\mathrm{CF}+$ Demean $=\mathrm{CF}$ with demeaned observed variables; $\mathrm{CF}+$ Demean $+\mathrm{PS}=\mathrm{CF}$ with demeaned observed variables and propensity scores trained with demeaned variables.

We conducted a sensitivity analysis to investigate whether our conclusions about the ATE would be altered by bias from an individual-level unmeasured confounder $U_{i j}$. In particular, we were interested in examining whether the positive effect of taking an eighth-grade algebra course would be changed into a non-significant effect through a negative bias from $U_{i j}$. We computed a new treatment effect, $\tau^{\dagger}$, by using the formula from Hong and Raudenbush (2006): $\tau^{\dagger}=\hat{\tau}+\zeta\left(E\left[U_{i j} \mid Z_{i j}=1\right]-E\left[U_{i j} \mid Z_{i j}=0\right]\right)$. We set $\zeta$ to 2.991, which was the second largest estimated regression coefficient of the observed student-level covariates from the outcome regression model. We chose the second largest because it is unlikely that there is an unmeasured individual-level confounder that is as strong as the prior achievement score, which is typically considered to be the most important predictor of future achievement. Similarly, to specify $\left(E\left[U_{i j} \mid Z_{i j}=1\right]-E\left[U_{i j} \mid Z_{i j}=0\right]\right)$, we used the second largest treatment-minus-control difference in the student-level covariates, controlling for the other observed covariates; this was 0.096. Then, we used a negative bias of $-0.287=-2.991 \cdot 0.096$, and we computed the $95 \%$ confidence interval of the adjusted ATE $\tau^{\dagger}$ with the estimated standard errors obtained from Table 3.

The results of the sensitivity analysis are in Table 4. We focused on estimators that were shown to control cluster-level unmeasured effects in our simulation studies (i.e., DRCGEE, Marginal+FePS, Clustered+FePS, CF+FePS, CF+Demean, CF+Demean+PS). With the estimated values of $\zeta$ and $E\left[U_{i j} \mid Z_{i j}=1\right]-E\left[U_{i j} \mid Z_{i j}=0\right]$, none of the confidence intervals included 0 . Thus, across the six estimators, we are confident that there is a positive effect of 
Table 4: Sensitivity analyses of ATE estimates of taking an eighth-grade algebra course

\begin{tabular}{lc}
\hline & $95 \%$ Confidence Interval \\
\hline DRCGEE & $(1.55,3.84)$ \\
Marginal+FePS & $(0.29,2.94)$ \\
Clustered+FePS & $(0.63,4.23)$ \\
CF+FePS & $(0.85,3.65)$ \\
CF+Demean & $(1.96,3.91)$ \\
CF+Demean+PS & $(1.67,3.47)$ \\
\hline
\end{tabular}

Note: DRCGEE $=$ the doubly robust conditional generalized estimating equation estimator; Marginal = the marginal inverse propensity weighting estimator; Clustered = the clustered inverse propensity weighting estimator; RePS = propensity scores from random effects logistic regression; FePS = propensity scores from fixed effects logistic regression; $\mathrm{CF}=$ Causal Forests without any modifications; $\mathrm{CF}+\mathrm{RePS}$ $=\mathrm{CF}$ with propensity scores from random effects logistic regression; $\mathrm{CF}+\mathrm{FePS}=\mathrm{CF}$ with propensity scores from fixed effects logistic regression; $\mathrm{CF}+$ ClusterID $=\mathrm{CF}$ with the additional set of cluster dummies; $\mathrm{CF}+$ Demean $=\mathrm{CF}$ with demeaned observed variables; $\mathrm{CF}+$ Demean $+\mathrm{PS}=\mathrm{CF}$ with demeaned observed variables and propensity scores trained with demeaned variables.

taking an eighth-grade algebra course and that the treatment effect is robust to unmeasured student-level confounding.

\section{Discussion and Conclusions}

The goal of this paper was to provide simple modifications that enable Causal Forests to address cluster-level unmeasured confounders in multilevel observational data. We proposed five different modifications to Causal Forest to remove the impact of unmeasured cluster-level confounders and we compared the performance of each modified estimator with that of the DRCGEE estimator, the marginal IPW estimator, and the clustered IPW estimator. Through our simulation studies, we found that the modifications based on fixed effects logistic regression and demeaned variables coupled with demeaned propensity scores $(\mathrm{CF}+\mathrm{FePS}, \mathrm{CF}+$ Demean $+\mathrm{PS})$ showed the most promise in reducing bias from cluster-level unmeasured confounders. We also illustrated the use of our proposed methods on the ECLS-K data, showing that there is a positive effect of taking an 8th grade algebra course on math achievement scores.

Although the modified ML methods are useful in increasing the accuracy of treatment effect estimates in the presence of unmeasured cluster-level confounding, they must contend with the accuracy versus interpretability trade-off. Specifically, simpler, more transparent models like linear regression may be preferable to black-box models like Causal Forests because of their ease of use, simple interpretation, and straightforward computation. In contrast, black-box models like Causal Forests may be advantageous compared to simpler, more transparent models when the underlying relationships are complex and a more accurate estimate is desired. If the sole purpose of the study is to obtain the most accurate estimate of the treatment effect, blackbox models like the default Causal Forests or our modifications of Causal Forests with fixed effects propensity scores may be preferred. However, if interpretation of the methodology for the audience is of equal concern, simpler, more transparent methods may be preferred. The results from the black-box methods could be used as a means to verify the conclusions from the simpler methods.

Our study has several limitations that might impact the interpretation of our results. First, we did not investigate every possible data generating process encountered in multilevel observational data. In particular, we did not consider all possible combinations of the simulation parameters used in our study, and we did not consider more complex, multilevel data structures, 
such as three-level data and cross-classified data. We did not examine correlated pre-treatment covariates or distributions of unmeasured cluster-level confounders beyond the Normal and uniform distributions. Also, although we varied the size of the clusters and the number of clusters to understand the impact that the ratio of these numbers had on the performance of our methods, our total sample size was fixed to around 4,000 and our results may not characterize multilevel studies with much smaller total sample sizes. Second, we assumed SUTVA and did not consider possible spillover effects among individuals within clusters. Third, we only focused on simple tuning procedures of Causal Forests; we did not explore other, potentially more complex tuning parameters such as tree pruning and adding a penalty for imbalanced splits.

Despite these limitations, we believe that our simple modifications of Causal Forests can improve robustness against cluster-level unmeasured confounders in multilevel observational studies. Additionally, we conjecture that the lessons learned from our study of Causal Forests could be applied to make other ML methods more robust in the analysis of multilevel/hierarchical data with cluster-level unmeasured confounding. In particular,

1. Based on the performance of $\mathrm{CF}+\mathrm{FePS}$ and $\mathrm{CF}+$ Demean $+\mathrm{PS}$, using either fixed effects propensity scores or demeaned observed variables with propensity scores trained from demeaned variables show promise in making ML methods more robust to biases arising from cluster-level unmeasured confounders in multilevel observational studies.

2. Even if propensity scores are partially misspecified, using them as working models inside ML methods may potentially mitigate biases from unmeasured cluster-level confounders, especially compared to biases from non-ML IPW estimators that use the same misspecified propensity scores.

3. Based on the performance of $\mathrm{CF}+\mathrm{RePS}$, we recommend against using random effects models to modify ML methods. Random effect models are sensitive to the distribution of unmeasured cluster-level confounders and the ratio of the number of clusters to cluster size.

4. We recommend against adding cluster dummy variables inside ML methods unless the number of clusters is small compared to the size of the clusters.

Although these conjectures may not work for every ML method in causal inference or every multilevel datasets, we hope that our findings can serve as useful starting points for researchers to fine-tune ML-based causal inference methods when dealing with multilevel observational data with cluster-level unmeasured confounding.

\section{References}

Anderson, R., \& Chang, B. (2011). Mathematics course-taking in rural high schools. Journal of Research in Rural Education, 26(1). http://sites.psu.edu/jrre/wp-content/uploads/ sites/6347/2014/02/26-1.pdf

Arkhangelsky, D., \& Imbens, G. (2019). The role of the propensity score in fixed effect models. arXiv. https://doi.org/10.3386/w24814

Arpino, B., \& Cannas, M. (2016). Propensity score matching with clustered data. an application to the estimation of the impact of caesarean section on the apgar score. Statistics in Medicine, 35(12), 2074-2091. https://doi.org/10.1002/sim.6880

Arpino, B., \& Mealli, F. (2011). The specification of the propensity score in multilevel observational studies. Computational Statistics \& Data Analysis, 55(4), 1770-1780. https: //doi.org/10.1016/j.csda.2010.11.008 
Athey, S., \& Imbens, G. (2016). Recursive partitioning for heterogeneous causal effects. Proceedings of the National Academy of Sciences, 113(27), 7353-7360. https://doi.org/10. 1073/pnas.1510489113

Athey, S., Tibshirani, J., \& Wager, S. (2019). Generalized random forests. The Annals of Statistics, 47(2), 1148-1178. https://doi.org/10.1214/18-AOS1709

Athey, S., \& Wager, S. (2019). Estimating treatment effects with causal forests: An application. Observational Studies, 5(2), 37-51. https://doi.org/10.1353/obs.2019.0001

Bates, D., Mächler, M., Bolker, B., \& Walker, S. (2015). Fitting linear mixed-effects models using lme4. Journal of Statistical Software, 67(1), 1-48. https://doi.org/10.18637/jss.v067.i01

Caliendo, M., \& Kopeinig, S. (2008). Some practical guidance for the implementation of propensity score matching. Journal of Economic Surveys, 22(1), 31-72. https://doi.org/10. 1111/j.1467-6419.2007.00527.x

Cogan, L. S., Schmidt, W. H., \& Wiley, D. E. (2001). Who takes what math and in which track? using TIMSS to characterize us students' eighth-grade mathematics learning opportunities. Educational Evaluation and Policy Analysis, 23(4), 323-341. https://doi.org/10. $3102 / 01623737023004323$

Dorie, V., Hill, J., Shalit, U., Scott, M., \& Cervone, D. (2019). Automated versus do-it-yourself methods for causal inference: Lessons learned from a data analysis competition. Statistical Science, 34(1), 43-68. https://doi.org/10.1214/18-STS667

Firebaugh, G., Warner, C., \& Massoglia, M. (2013). Fixed effects, random effects, and hybrid models for causal analysis (S. L. Morgan, Ed.). In S. L. Morgan (Ed.), Handbook of causal analysis for social research. Springer. https://doi.org/10.1007/978-94-007-6094-3_7

Goetgeluk, S., \& Vansteelandt, S. (2008). Conditional generalized estimating equations for the analysis of clustered and longitudinal data. Biometrics, 64 (3), 772-780. https://doi.org/ 10.1111/j.1541-0420.2007.00944.x

Hansen, L. P. (1982). Large sample properties of generalized method of moments estimators. Econometrica: Journal of the Econometric Society, 1029-1054. https://doi.org/10.2307/ 1912775

He, Z. (2018). Inverse conditional probability weighting with clustered data in causal inference. arXiv. https://arxiv.org/abs/1808.01647

Hernan, M. A., \& Robins, J. M. (2020). Causal inference: What if. Boca Raton: Chapman \& Hall/CRC. https://cdn1.sph.harvard.edu/wp- content/uploads/sites / 1268/2021/03/ ciwhatif_hernanrobins_30mar21.pdf

Hill, J. L. (2011). Bayesian nonparametric modeling for causal inference. Journal of Computational and Graphical Statistics, 20(1), 217-240. https://doi.org/10.1198/jcgs.2010.08162

Hong, G., \& Raudenbush, S. W. (2006). Evaluating kindergarten retention policy: A case study of causal inference for multilevel observational data. Journal of the American Statistical Association, 101, 901-910. https://doi.org/10.1198/016214506000000447

Hong, G., \& Raudenbush, S. W. (2013). Heterogeneous agents, social interactions, and causal inference (S. L. Morgan, Ed.). In S. L. Morgan (Ed.), Handbook of causal analysis for social research. Springer. https://doi.org/10.1007/978-94-007-6094-3_16

Imai, K., \& Kim, I. S. (2019). When should we use unit fixed effects regression models for causal inference with longitudinal data? American Journal of Political Science, 63(2), 467-490. https://doi.org/10.1111/ajps.12417

Imai, K., \& Ratkovic, M. (2013). Estimating treatment effect heterogeneity in randomized program evaluation. The Annals of Applied Statistics, 7(1), 443-470. https://doi.org/10. 1214/12-AOAS593

Imbens, G. W., \& Rubin, D. B. (2015). Causal inference in statistics, social, and biomedical sciences. Cambridge University Press. https://doi.org/10.1017/CBO9781139025751 
Kang, J. D. Y., \& Schafer, J. L. (2007). Demystifying double robustness: A comparison of alternative strategies for estimating a population mean from incomplete data. Statistical Science, 22(4), 523-539. https://doi.org/10.1214/07-STS227

Kennedy, E. H. (2020). Optimal doubly robust estimation of heterogeneous causal effects. arXiv. https://arxiv.org/abs/2004.14497

Kim, J.-S., \& Steiner, P. M. (2015). Multilevel propensity score methods for estimating causal effects: A latent class modeling strategy. In L. . van der Ark, D. M. Bolt, W.-C. Wang, J. A. Douglas, \& M. Wiberg (Eds.), Quantitative psychology research: The 80th annual meeting of the psychometric society (pp. 293-306). Springer. https://doi.org/10.1007/ 978-3-319-19977-1_21

Kim, J., \& Seltzer, M. (2007). Causal inference in multilevel settings in which selection processes vary across schools (working paper 708). University of California, Los Angeles, Center for the Study of Evaluation.

Kim, Y., \& Steiner, P. M. (2020). Causal graphical views of fixed effects and random effects models. The British Journal of Mathematical and Statistical Psychology, 74 (2), 165-183. https://doi.org/10.1111/bmsp.12217

Künzel, S. R., Sekhon, J. S., Bickel, P. J., \& Yu, B. (2019). Metalearners for estimating heterogeneous treatment effects using machine learning. Proceedings of the National Academy of Sciences, 116(10), 4156-4165. https://doi.org/10.1073/pnas.1804597116

Lee, Y., Nguyen, T. Q., \& Stuart, E. A. (2021). Partially pooled propensity score models for average treatment effect estimation with multilevel data. Journal of the Royal Statistical Society: Series A (Statistics in Society). https://doi.org/10.1111/rssa.12741

Leite, W. L., Jimenez, F., Kaya, Y., Stapleton, L. M., MacInnes, J. W., \& Sandbach, R. (2015). An evaluation of weighting methods based on propensity scores to reduce selection bias in multilevel observational studies. Multivariate Behavioral Research, 50(3), 265-284. https://doi.org/10.1080/00273171.2014.991018

Leite, W. L. (2016). Practical propensity score methods using R. Sage Publications. https://doi. org $/ 10.4135 / 9781071802854$

Li, F., Zaslavsky, A. M., \& Landrum, M. B. (2013). Propensity score weighting with multilevel data. Statistics in Medicine, 32(19), 3373-3387. https://doi.org/10.1002/sim.5786

Li, Y., Lee, Y., Port, F. K., \& Robinson, B. M. (2019). The impact of unmeasured within-and between-cluster confounding on the bias of effect estimators of a continuous exposure. Statistical Methods in Medical Research, (8), 2119-2139. https://doi.org/10.1177/ 0962280219883323

Lunceford, J. K., \& Davidian, M. (2004). Stratification and weighting via the propensity score in estimation of causal treatment effects: A comparative study. Statistics in Medicine, 23(19), 2937-2960.

Neyman, J. S. (1923). On the application of probability theory to agricultural experiments: Essay on principles. section 9 (with discussion). Statistical Science, 4, 465-480.

Nie, X., \& Wager, S. (2020). Quasi-oracle estimation of heterogeneous treatment effects. Biometrika, 108(2), 299-319. https://doi.org/10.1093/biomet/asaa076

Opdenakker, M.-C., \& Van Damme, J. (2001). Relationship between school composition and characteristics of school process and their effect on mathematics achievement. British Educational Research Journal, 27(4), 407-432. https://doi.org/10.1080/01411920120071434

$\mathrm{R}$ Core Team. (2020). R: A language and environment for statistical computing. R Foundation for Statistical Computing. Vienna, Austria. https://www.R-project.org/

Raudenbush, S. W., \& Bryk, A. S. (2002). Hierarchical linear models: Applications and data analysis methods. Sage.

Rickles, J. H. (2013). Examining heterogeneity in the effect of taking algebra in eighth grade. The Journal of Educational Research, 106(4), 251-268. https://doi.org/10.1080/00220671. 2012.692731 
Rickles, J. H., \& Seltzer, M. (2014). A two-stage propensity score matching strategy for treatment effect estimation in a multisite observational study. Journal of Educational and Behavioral Statistics, 39(6), 612-636. https://doi.org/10.3102/1076998614559748

Robins, J. M., Mark, S. D., \& Newey, W. K. (1992). Estimating exposure effects by modelling the expectation of exposure conditional on confounders. Biometrics, 479-495. https: //doi.org/10.2307/2532304

Rosenbaum, P. R., \& Rubin, D. B. (1983). The central role of the propensity score in observational studies for causal effects. Biometrika, 70, 41-55. https://doi.org/10.1093/biomet/ 70.1.41

Rubin, D. B. (1974). Estimating causal effects of treatments in randomized and nonrandomized studies. Journal of Educational Psychology, 66(5), 688-701. https://doi.org/10.1037/ h0037350

Rubin, D. B., \& Thomas, N. (1992). Characterizing the effect of matching using linear propensity score methods with normal distributions. Biometrika, 79(4), 797-809. https://doi.org/ 10.1093/biomet/79.4.797

Schafer, J. L., \& Kang, J. (2008). Average causal effects from nonrandomized studies: A practical guide and simulated example. Psychological Methods, 13(4), 279. https://doi.org/10. $1037 / \mathrm{a} 0014268$

Schuler, M. S., Chu, W., \& Coffman, D. (2016). Propensity score weighting for a continuous exposure with multilevel data. Health Services and Outcomes Research Methodology, 16(4), 271-292. https://doi.org/10.1007/s10742-016-0157-5

Snijders, T. A. B., \& Bosker, R. J. (2012). Multilevel analysis: An introduction to basic and advanced multilevel modeling (2nd ed.). Sage.

Steiner, P. M., Kim, J.-S., \& Thoemmes, F. (2012). Matching strategies for observational multilevel data, In Joint Statistical Meeting Proceedings, San Diego, California, American Statistical Association.

Stuart, E. A. (2010). Matching methods for causal inference: A review and a look forward. Statistical Science, 25(1), 1-21. https://doi.org/10.1214/09-STS313

Su, X., Tsai, C.-L., Wang, H., Nickerson, D. M., \& Li, B. (2009). Subgroup analysis via recursive partitioning. Journal of Machine Learning Research, 10(2), 141-158. https://doi.org/ $10.2139 /$ ssrn.1341380

Suk, Y., \& Kang, H. (2020). Robust estimation of causal effects in multilevel observational studies under arbitrary cluster-level unmeasured confounding. PsyArXiv. https://doi. org/10.31234/osf.io/t7vbz

Suk, Y., Kang, H., \& Kim, J.-S. (2020). Random forests approach for causal inference with clustered observational data. Multivariate Behavioral Research, 0(0), 1-24. https://doi. org/10.1080/00273171.2020.1808437

Thoemmes, F. J., \& West, S. G. (2011). The use of propensity scores for nonrandomized designs with clustered data. Multivariate Behavioral Research, 46(3), 514-543. https://doi.org/ 10.1080/00273171.2011.569395

Tibshirani, J., Athey, S., \& Wager, S. (2020). Grf: Generalized random forests [R package version 1.2.0]. R package version 1.2.0. https://CRAN.R-project.org/package=grf

Tourangeau, K., Le, T., Nord, C., Sorongon, A. G., \& Chapman, C. Early childhood longitudinal study, kindergarten class of 1998-99 (ECLS-K): Eighth-grade methodology report. 2009. https://nces.ed.gov/pubs2009/2009003.pdf

Wager, S., \& Athey, S. (2018). Estimation and inference of heterogeneous treatment effects using random forests. Journal of the American Statistical Association, 113(523), 1228-1242. https://doi.org/10.1080/01621459.2017.1319839

Walston, J., \& McCarroll, J. C. (2010). Eighth-grade algebra: Findings from the eighth-grade round of the early childhood longitudinal study, kindergarten class of 1998-99 (ECLS-K). statistics in brief. NCES 2010-016. National Center for Education Statistics. 
Wooldridge, J. M. (2010). Econometric analysis of cross section and panel data. MIT press.

Yang, S. (2018). Propensity score weighting for causal inference with clustered data. Journal of Causal Inference, 6(2). https://doi.org/10.1515/jci-2017-0027

Zetterqvist, J., \& Sjölander, A. (2015). Doubly robust estimation with the R package drgee. Epidemiologic Methods, 4(1), 69-86. https://doi.org/10.1515/em-2014-0021

Zetterqvist, J., Vansteelandt, S., Pawitan, Y., \& Sjölander, A. (2016). Doubly robust methods for handling confounding by cluster. Biostatistics, 17(2), 264-276. https://doi.org/10. 1093/biostatistics/kxv041 
Supplemental Material for Youmi Suk and Hyunseung Kang, Tuning random forests for causal inference under cluster-level unmeasured confounding. 


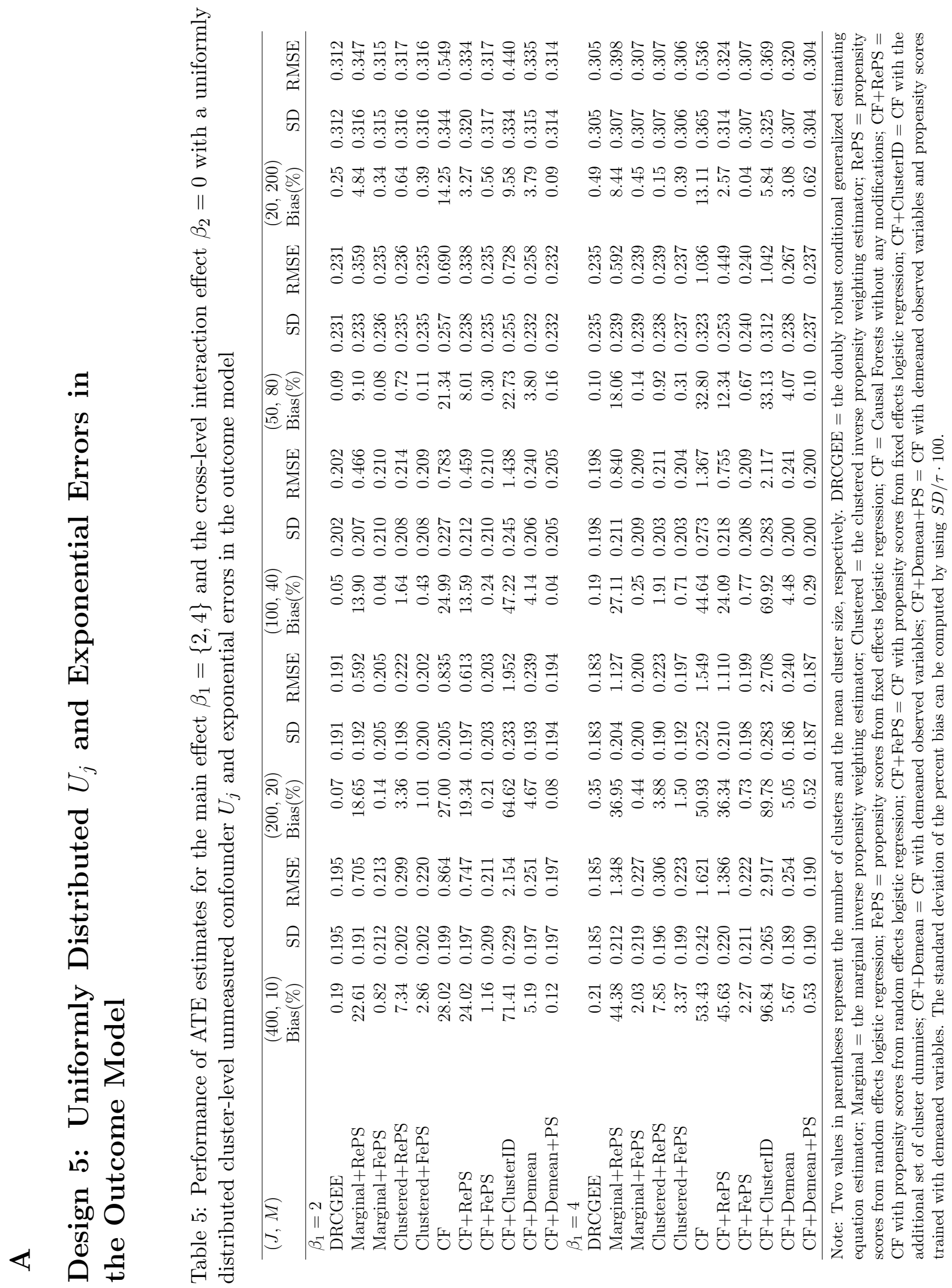




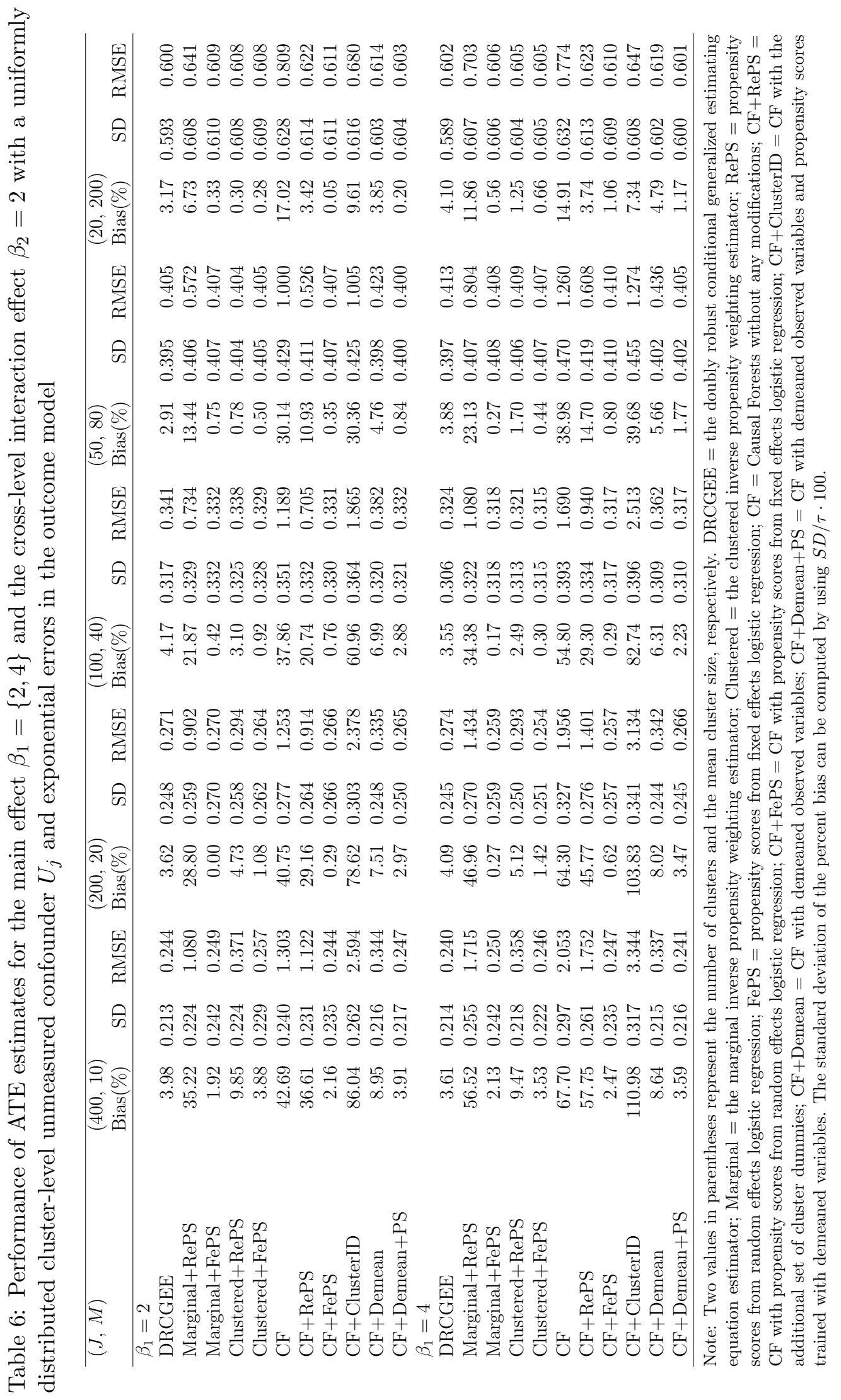




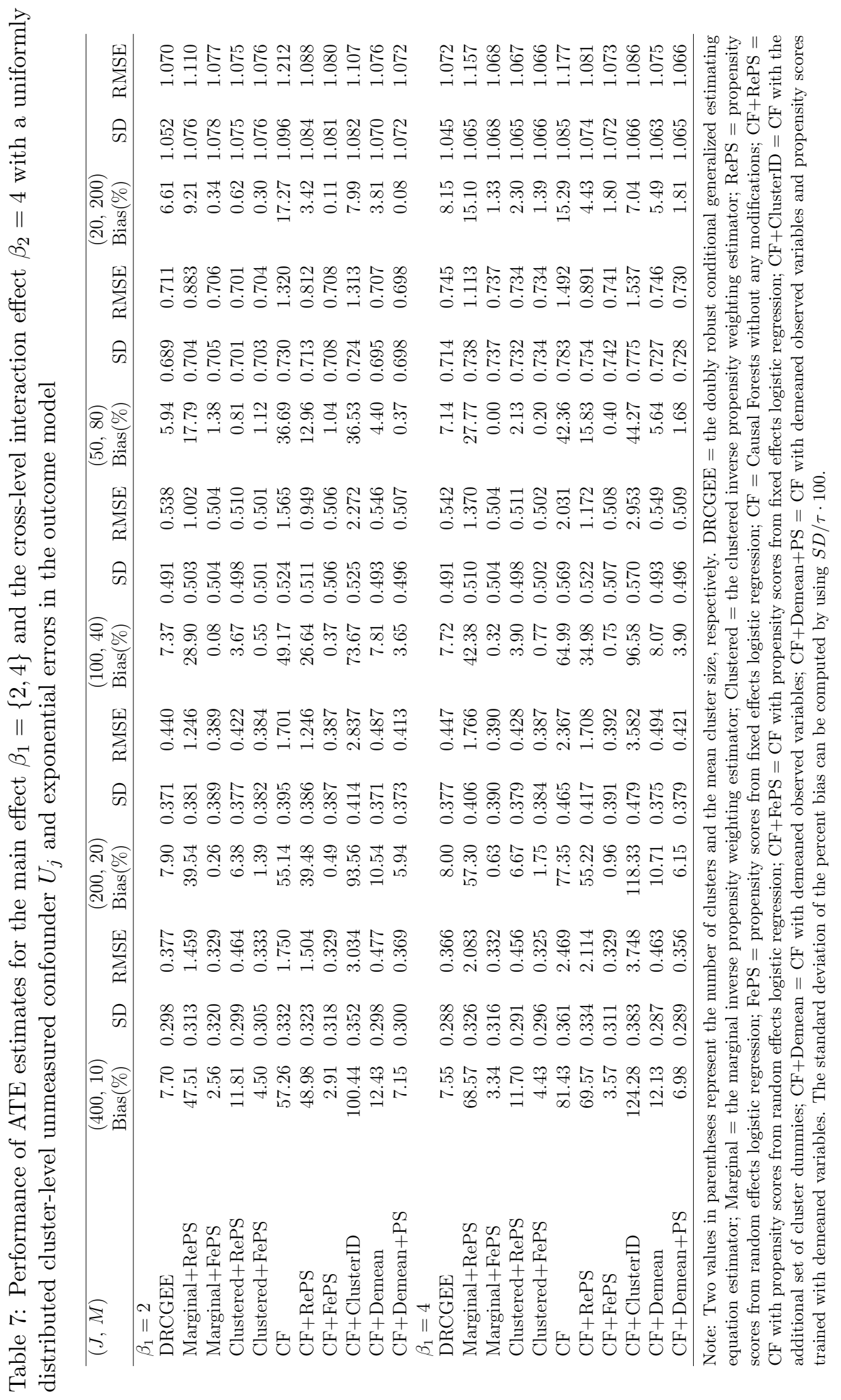




\section{Non-Machine-Learning Methods}
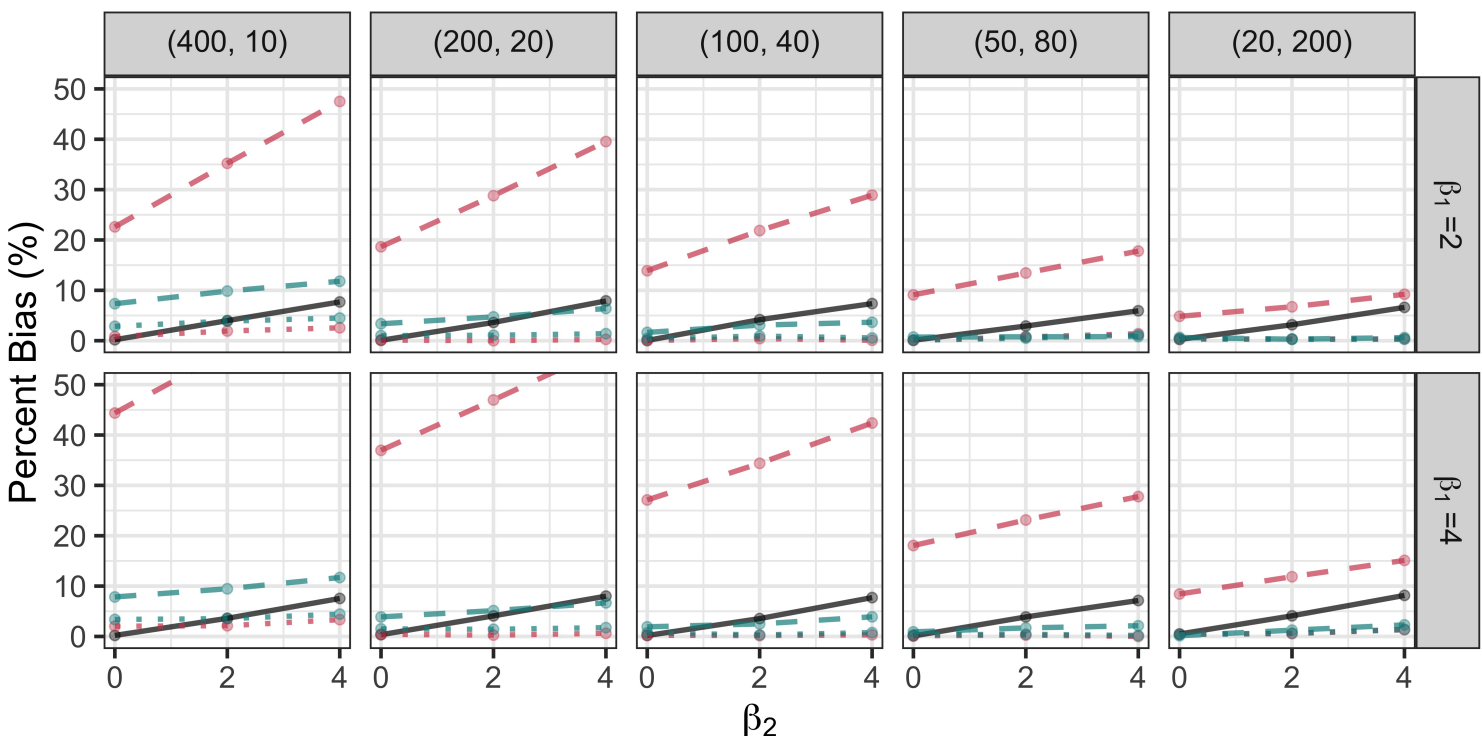

Estimator - DRCGEE $\rightarrow$ Marginal+RePS $: \circ$ Marginal+FePS $\Rightarrow$ Clustered+RePS $=$ : Clustered+FePS

\section{Machine Learning Methods}
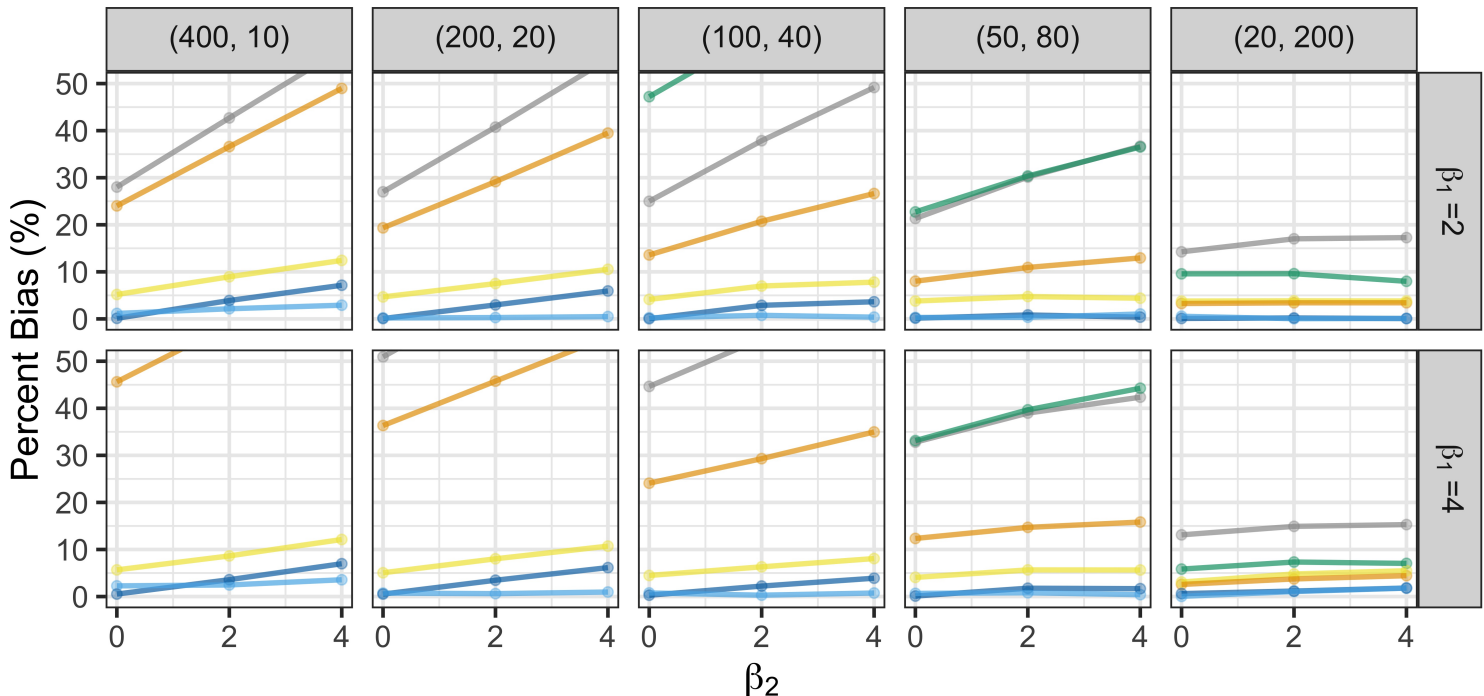

$$
\begin{aligned}
\text { Estimator } & -\mathrm{CF}=\mathrm{CF}+\mathrm{RePS}=\mathrm{CF}+\mathrm{FePS}=\mathrm{CF}+\text { ClusterID }-\mathrm{CF}+\text { Demean } \\
& -\mathrm{CF}+\text { Demean+PS }
\end{aligned}
$$

Figure 5: Performance of average treatment effect estimates in Design 5. The terms $\beta_{1}$ and $\beta_{2}$ represent the main effect term and the cross-level interaction effect term in the outcome model. Two values in the parentheses represent the number of clusters and the mean cluster size, respectively. All conditions for which percent bias exceeded $50 \%$ were omitted to permit clearer comparison of the less biased estimators. DRCGEE $=$ the doubly robust conditional generalized estimating equation estimator; Marginal $=$ the marginal inverse propensity weighting estimator; Clustered $=$ the clustered inverse propensity weighting estimator; RePS = propensity scores from random effects logistic regression; FePS = propensity scores from fixed effects logistic regression; $\mathrm{CF}=$ Causal Forests without any modifications; $\mathrm{CF}+\mathrm{RePS}=\mathrm{CF}$ with propensity scores from random effects logistic regression; $\mathrm{CF}+\mathrm{FePS}=\mathrm{CF}$ with propensity scores from fixed effects logistic regression; $\mathrm{CF}+\mathrm{ClusterID}=\mathrm{CF}$ with the additional set of cluster dummies; $\mathrm{CF}+$ Demean $=\mathrm{CF}$ with demeaned observed variables; $\mathrm{CF}+$ Demean $+\mathrm{PS}=\mathrm{CF}$ with demeaned observed variables and propensity scores trained with demeaned variables. 


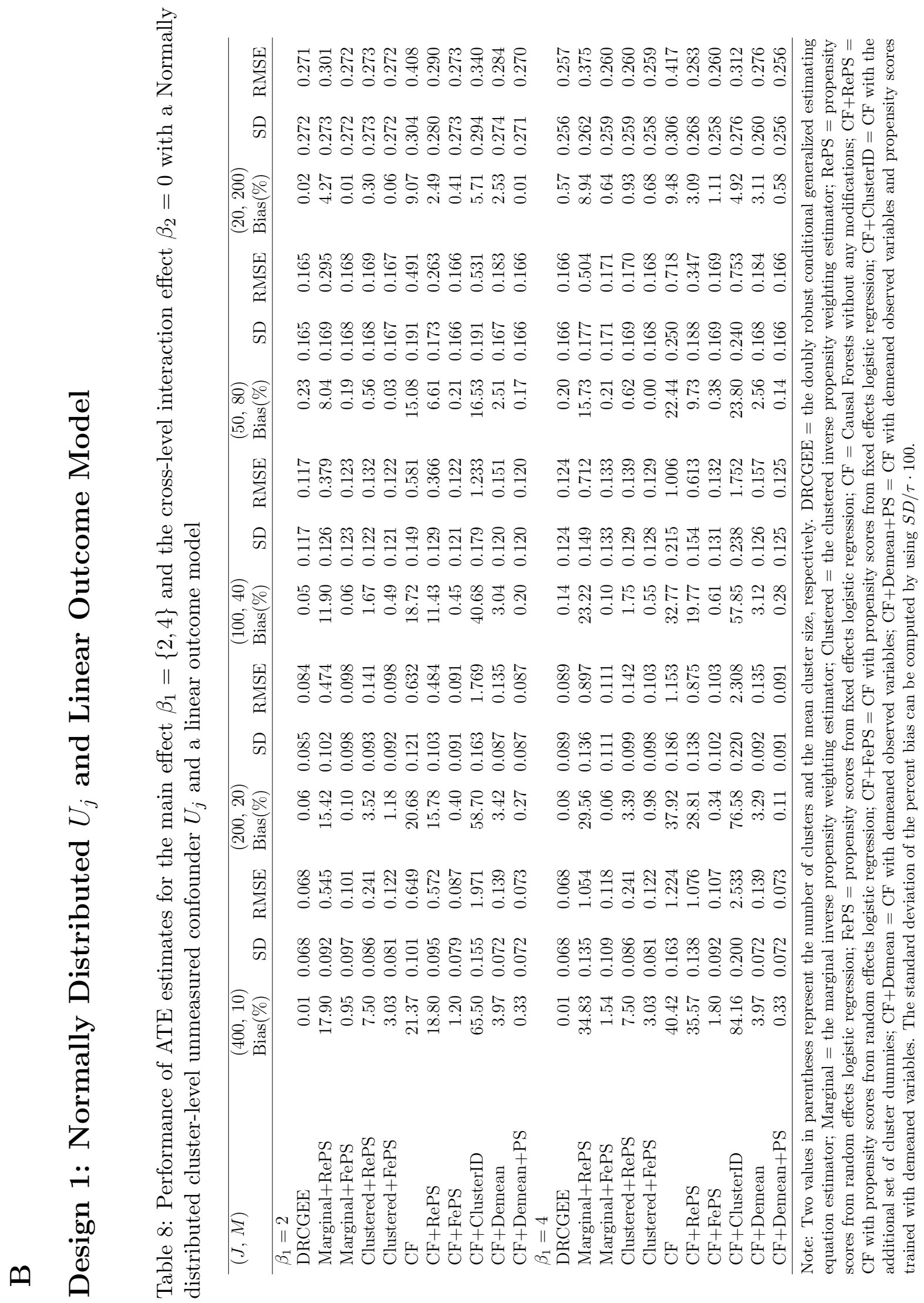




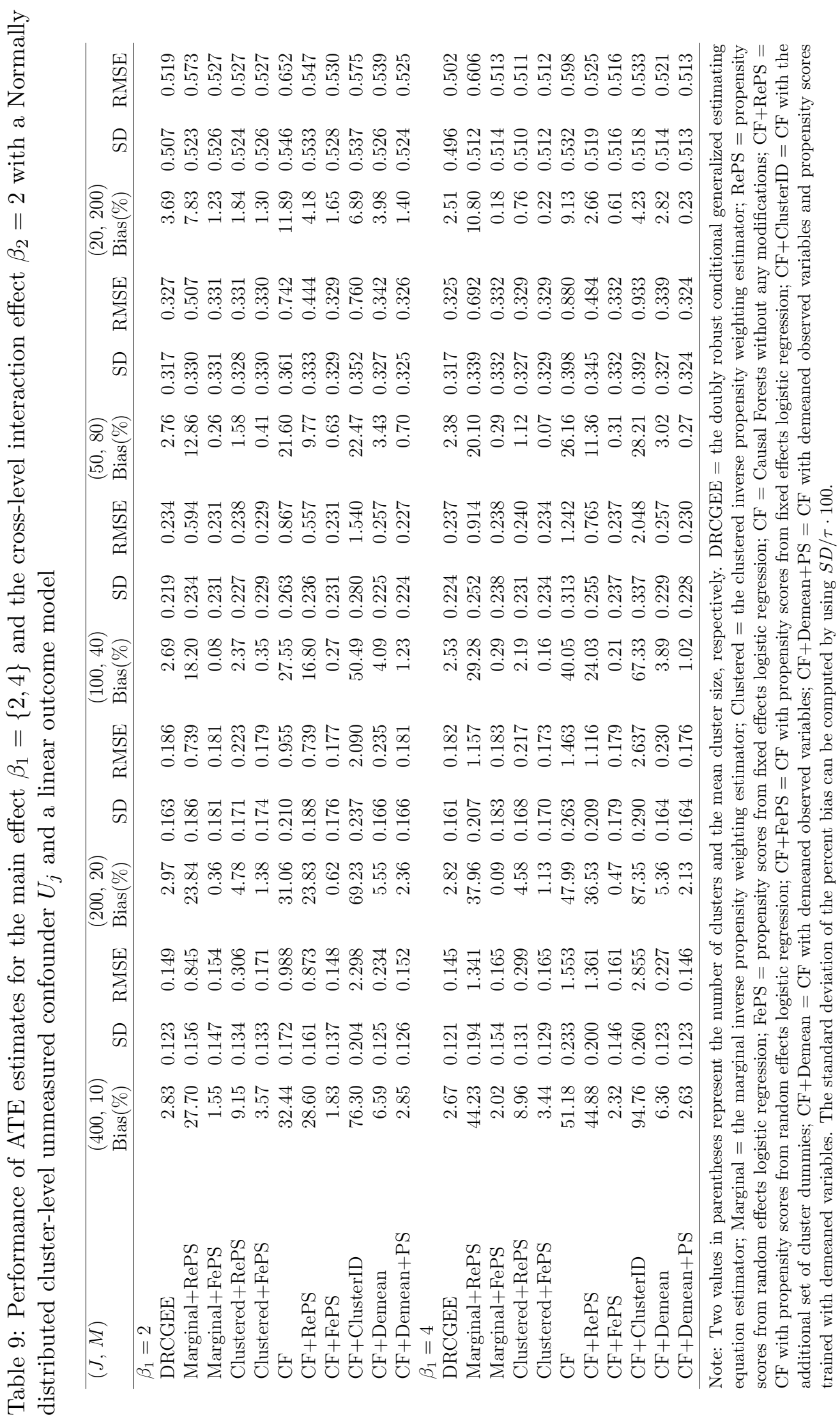




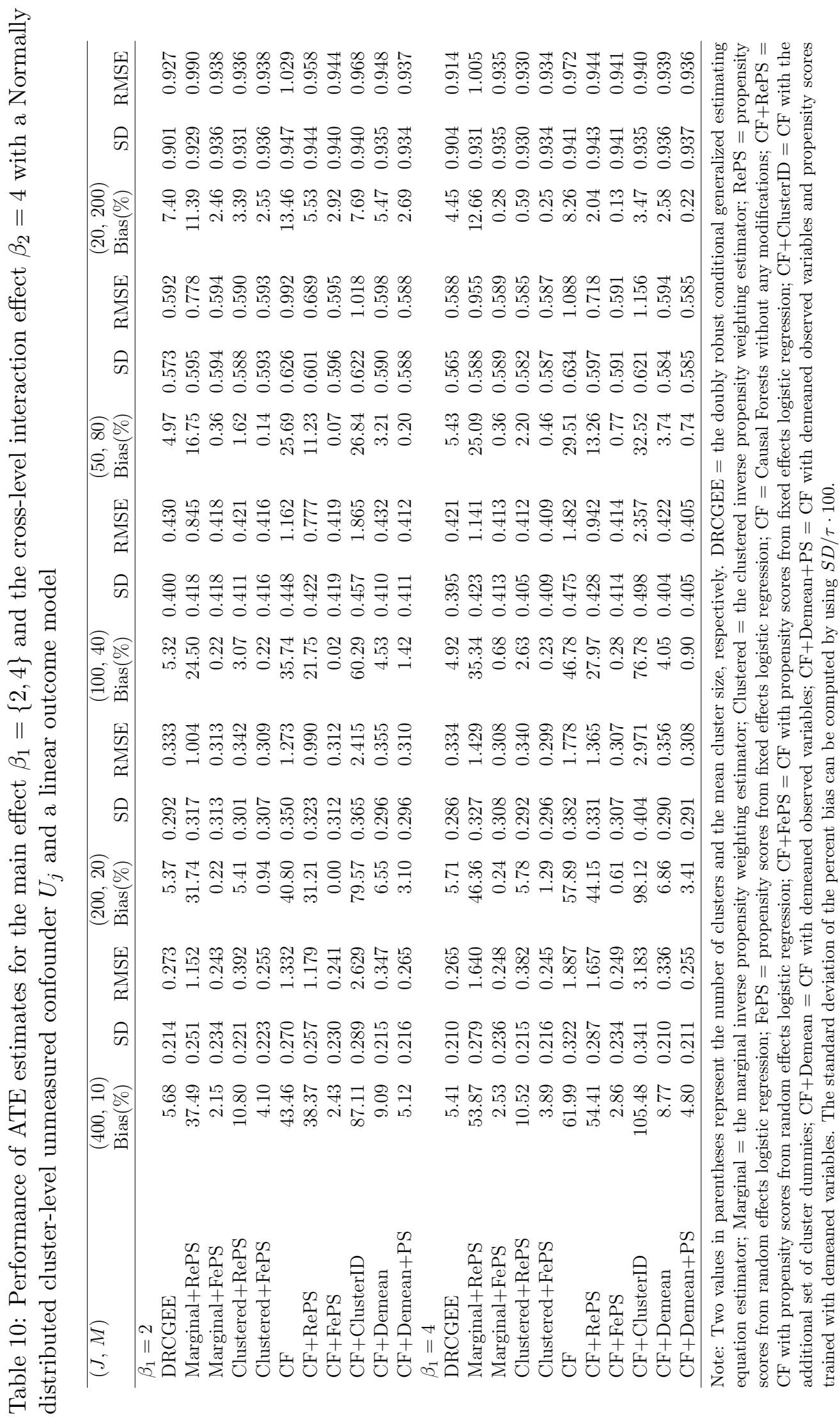




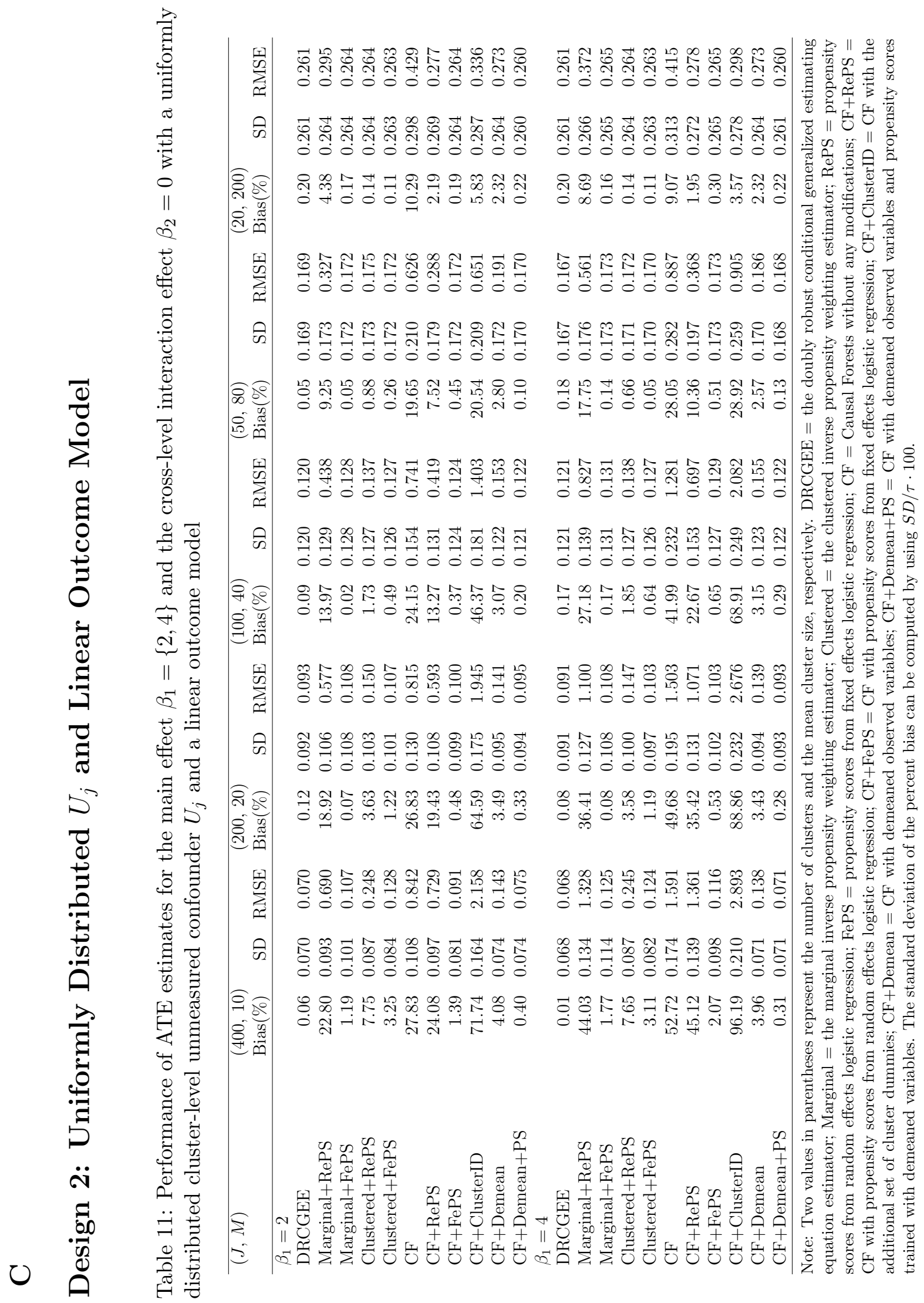




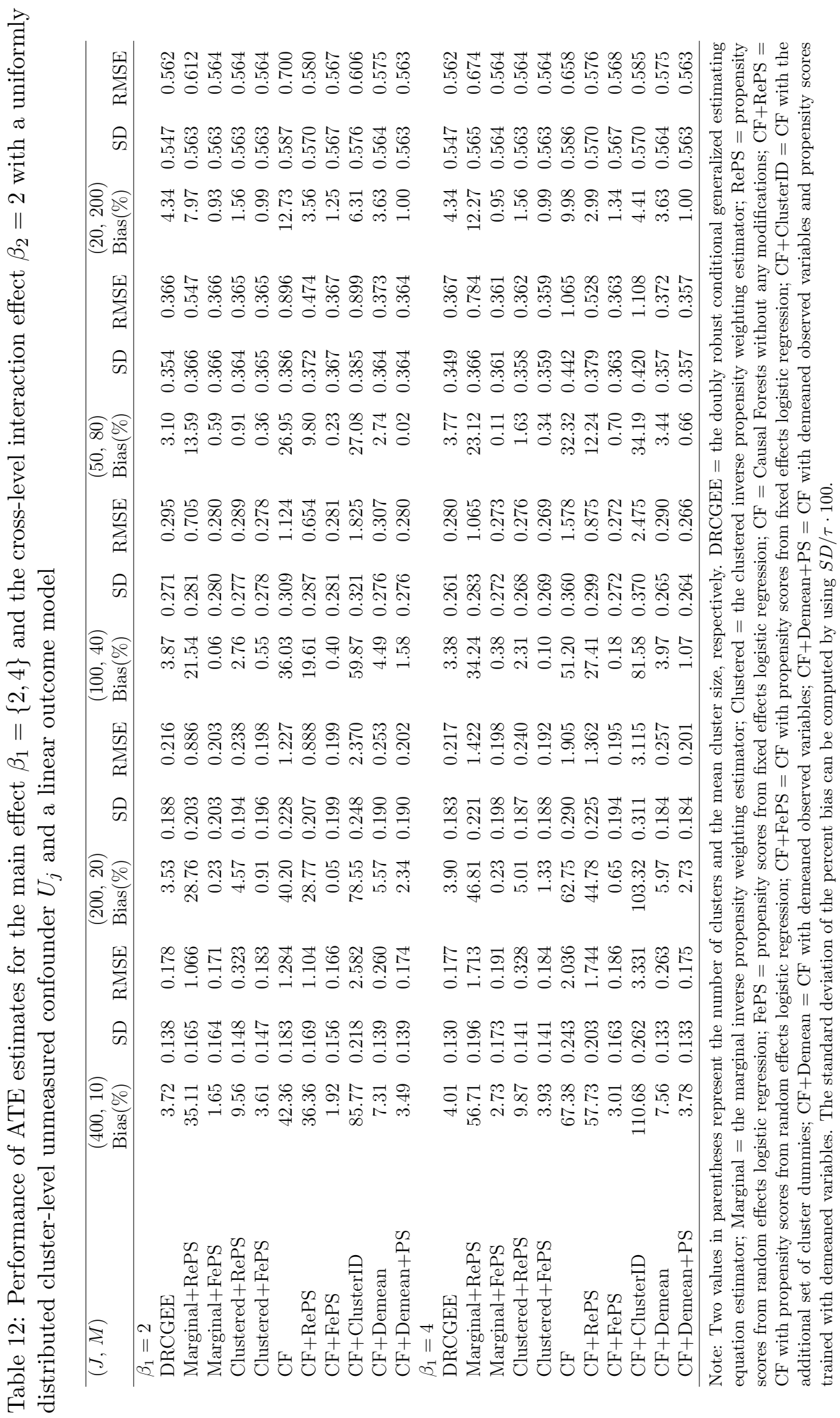




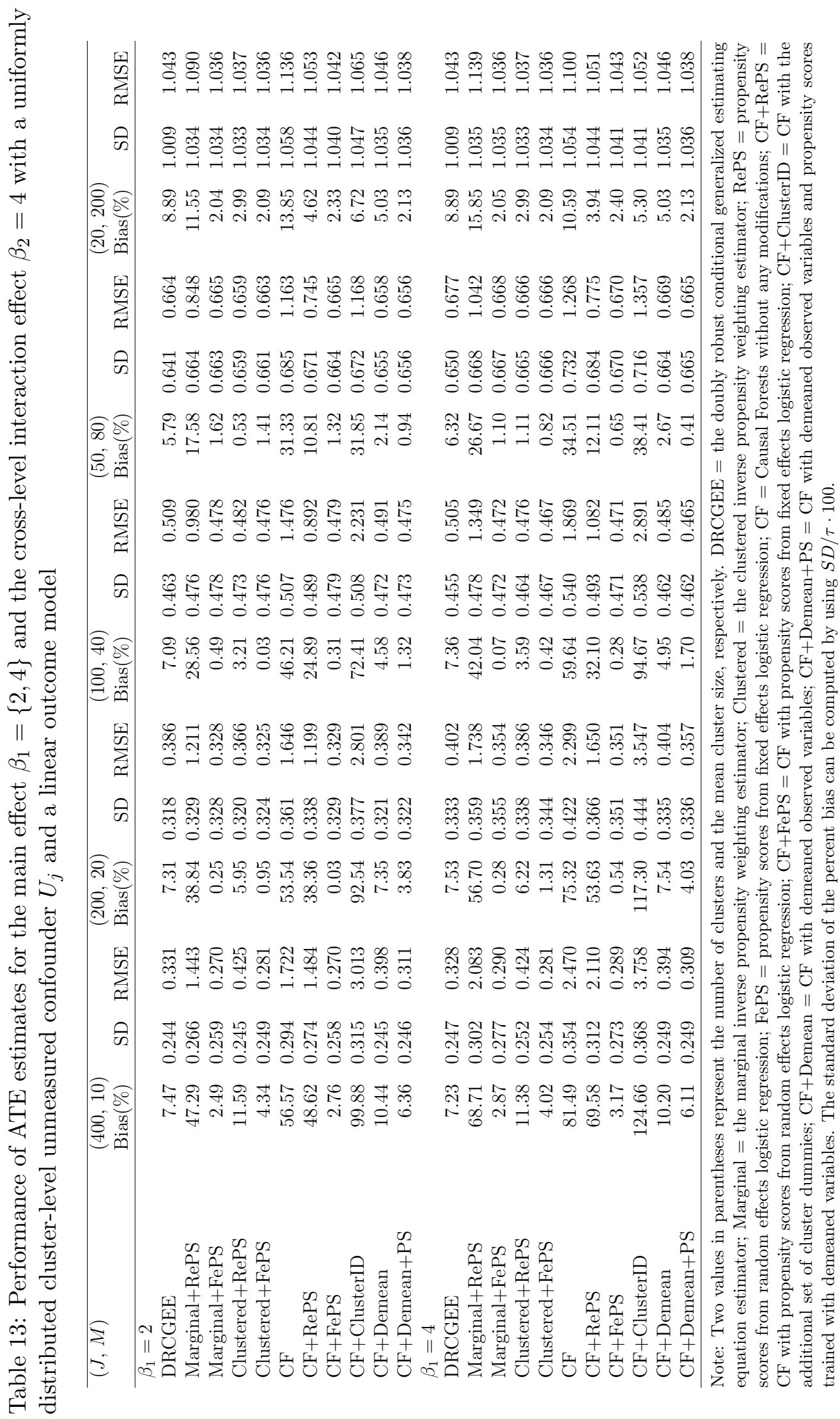




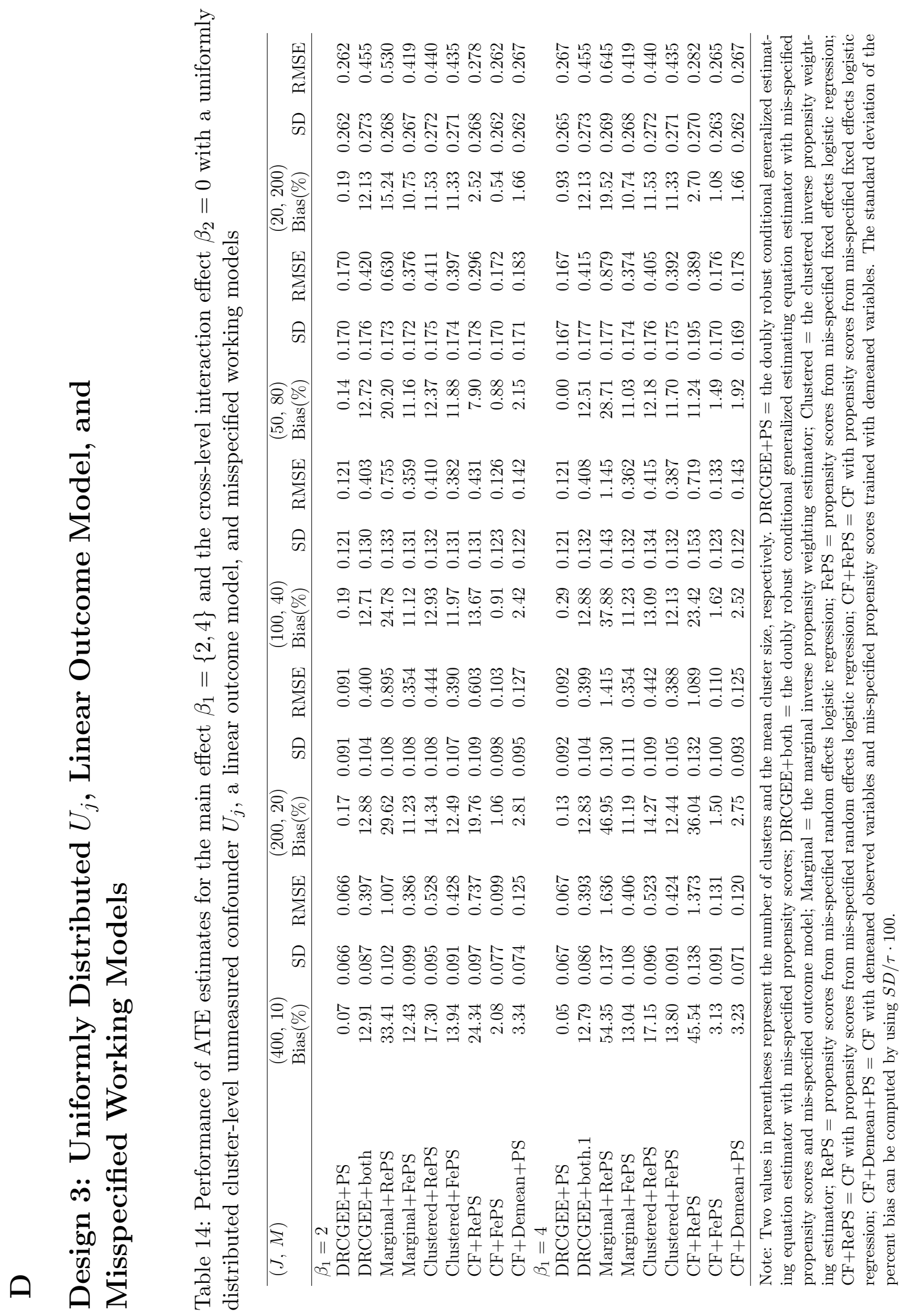




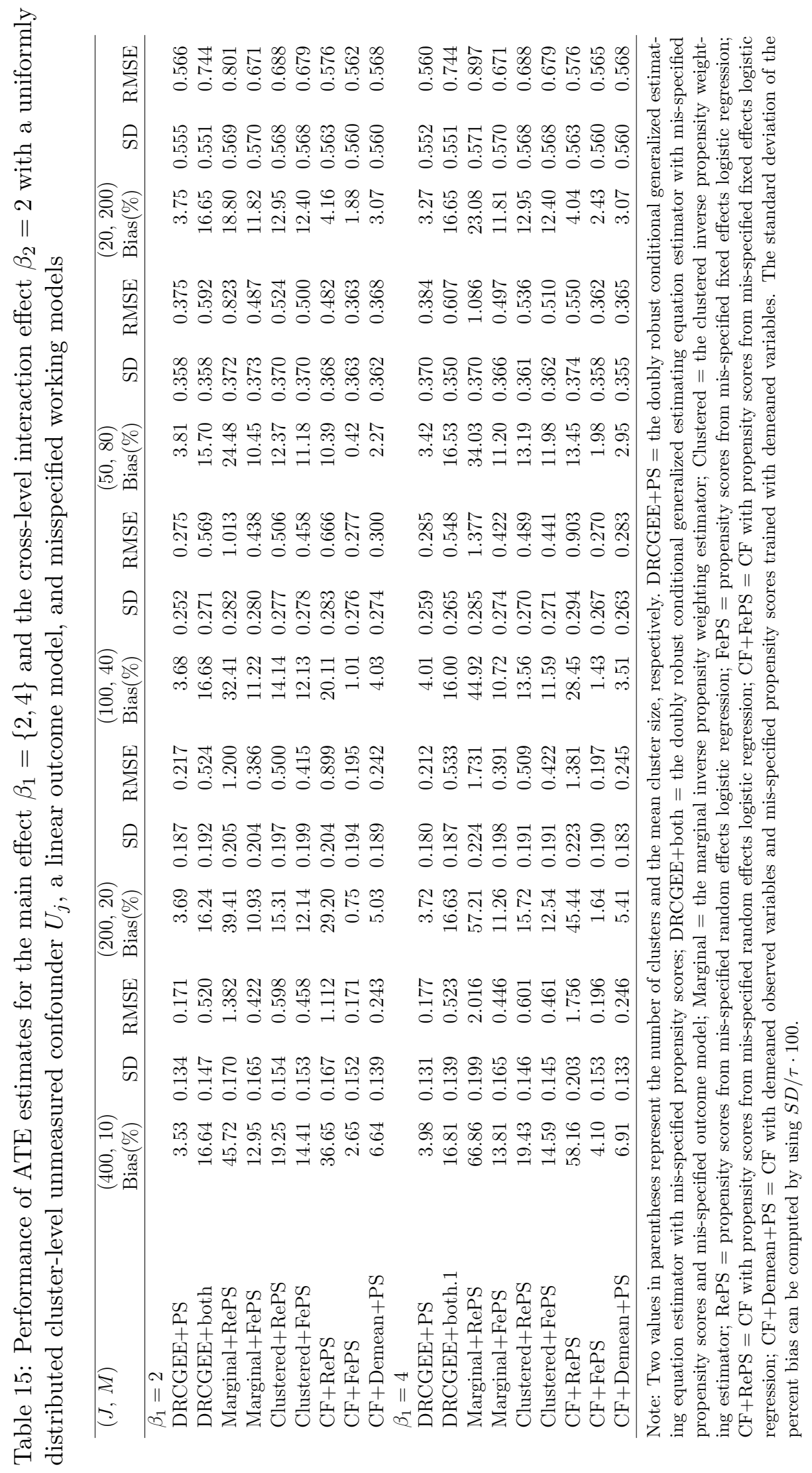




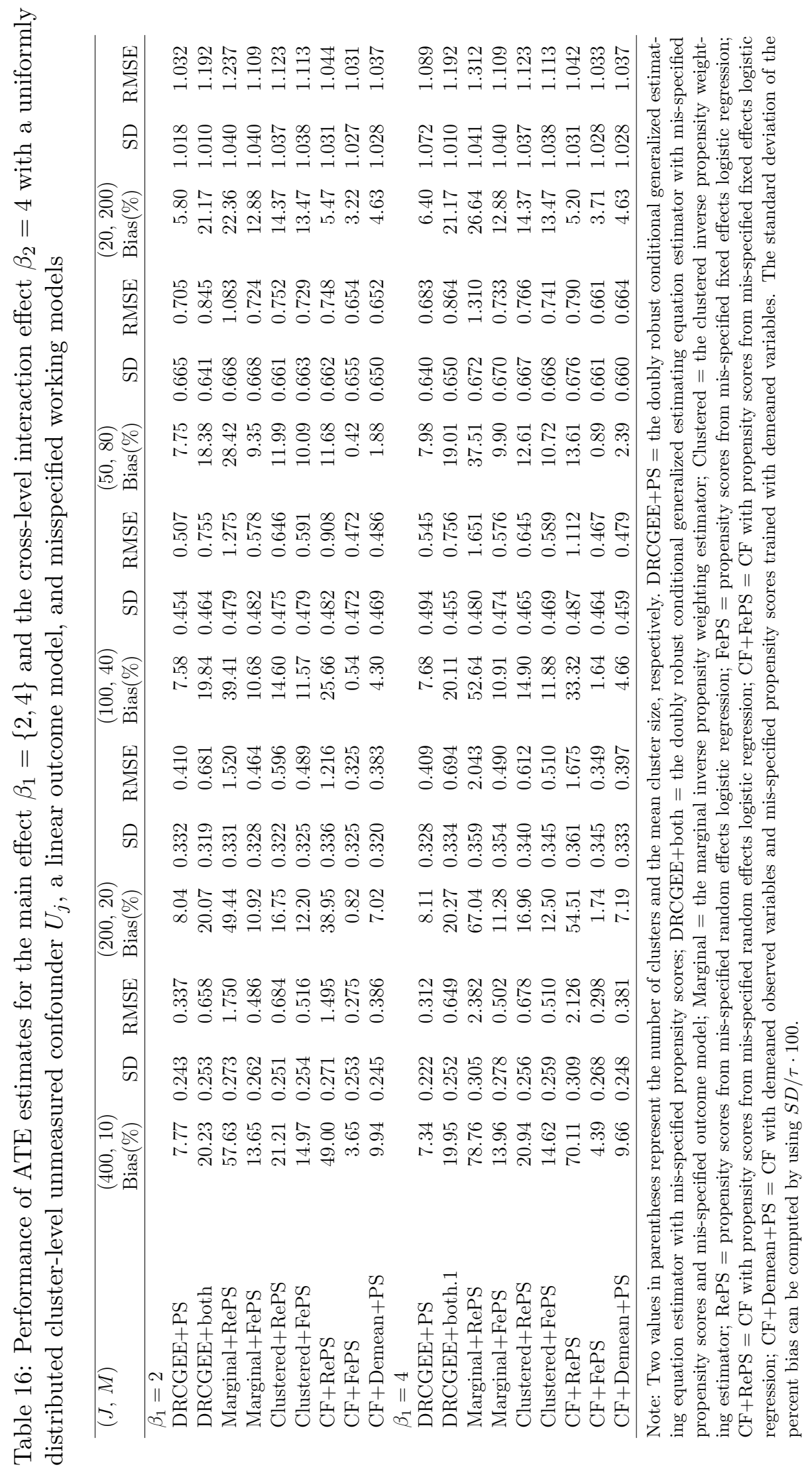




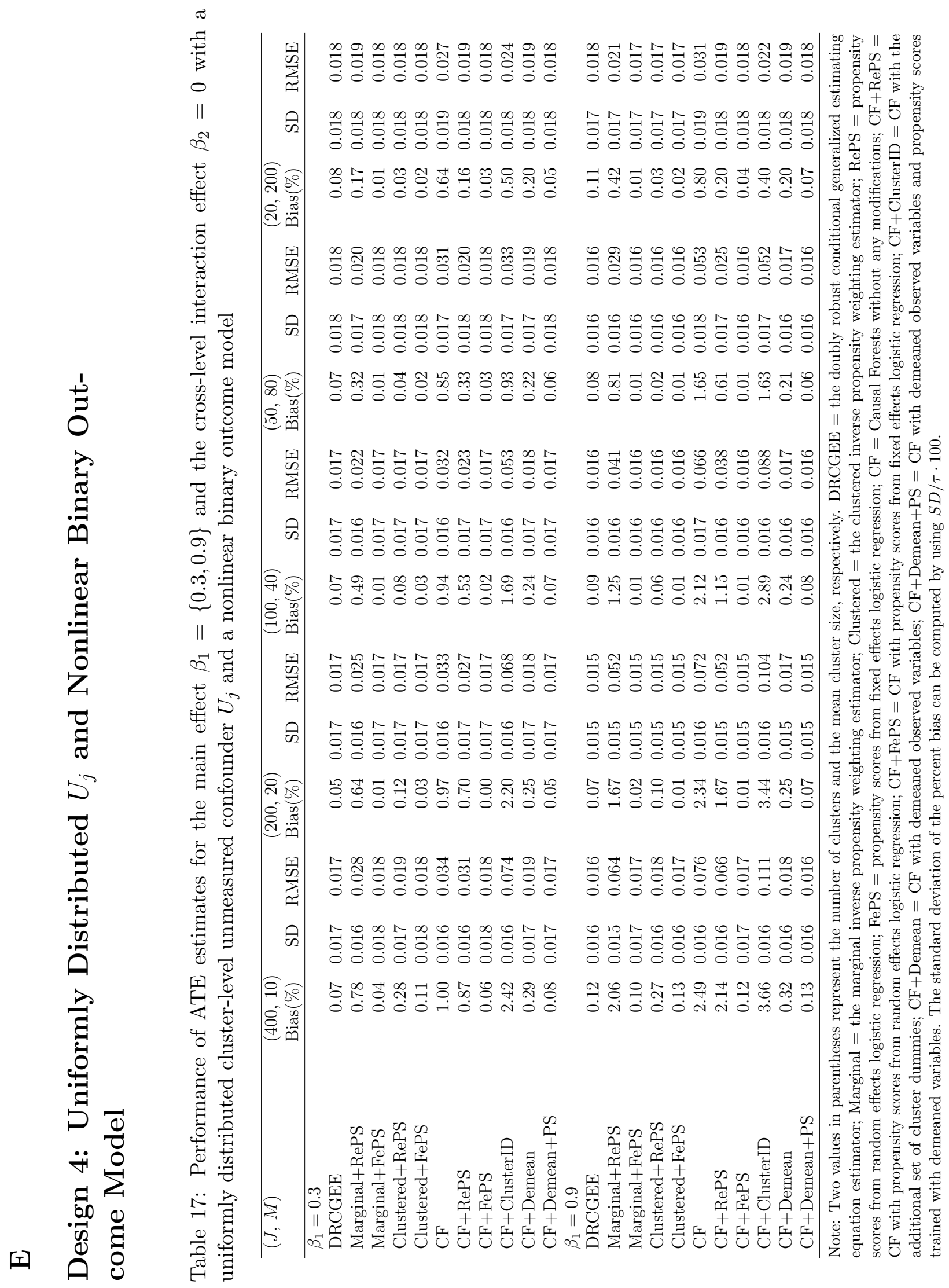




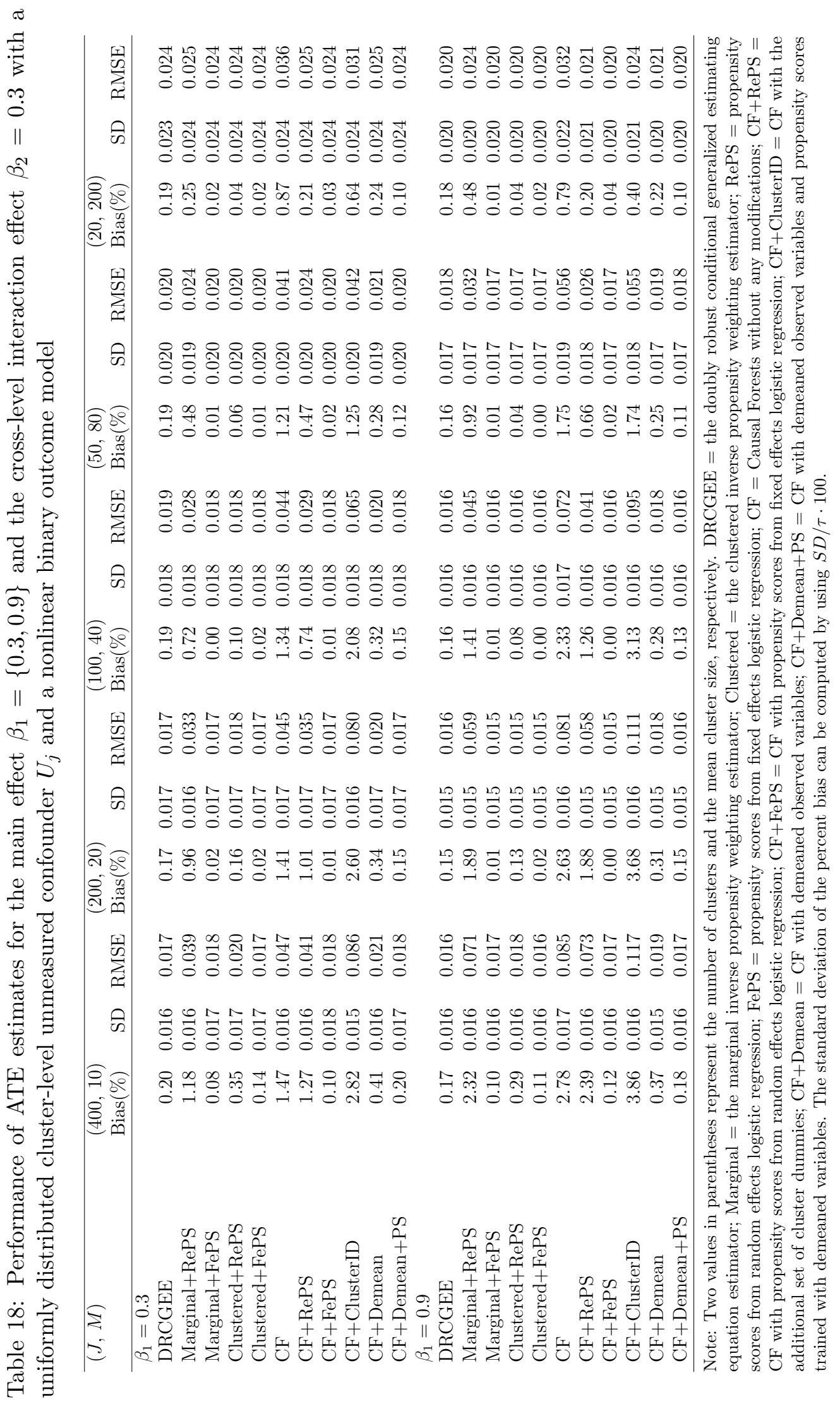




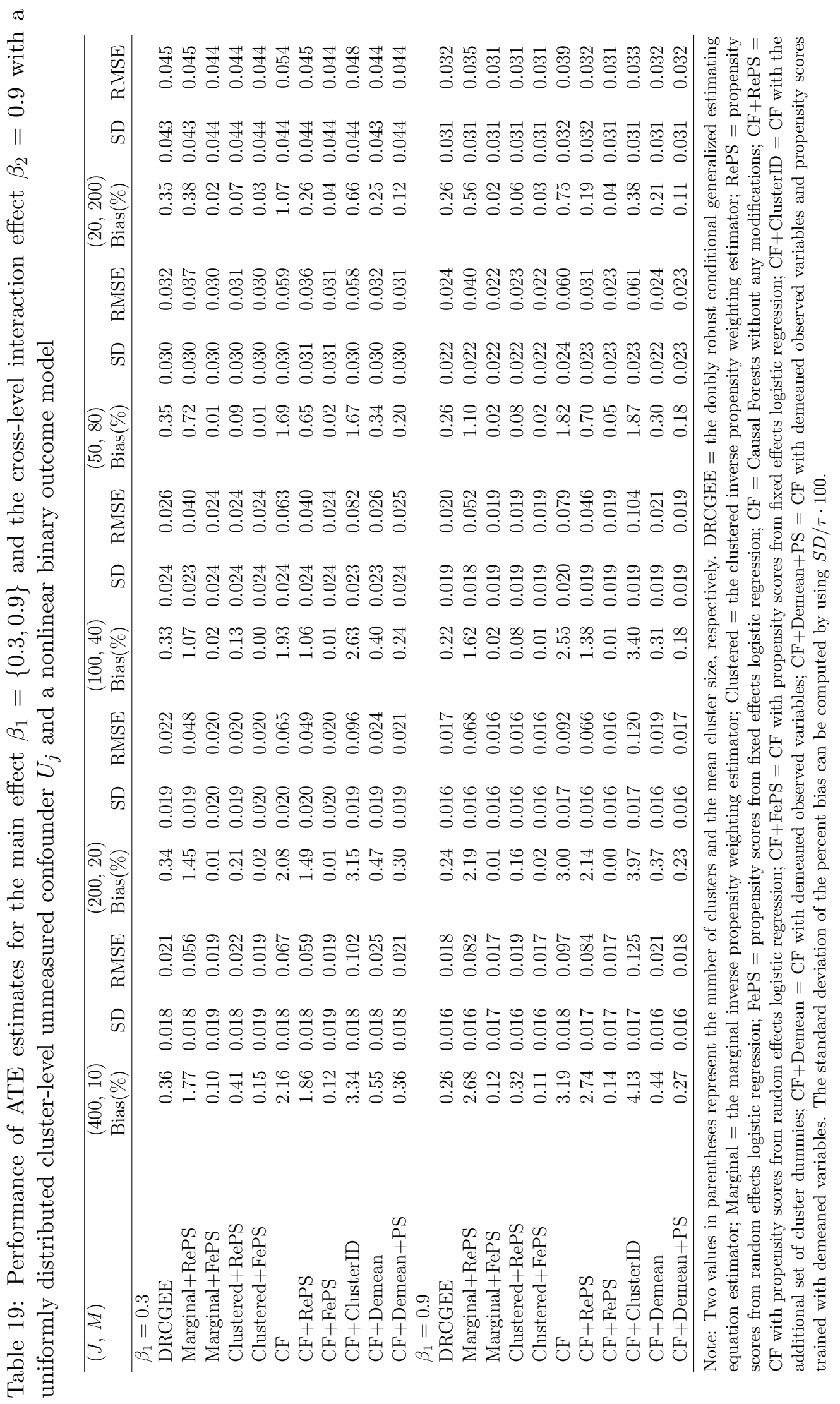

